\title{
Silencing SMOC2 ameliorates kidney fibrosis by inhibiting fibroblast to myofibroblast transformation
}

\author{
Casimiro Gerarduzzi, ${ }^{1}$ Ramya K. Kumar, ${ }^{1}$ Priyanka Trivedi,, ${ }^{1}$ Amrendra K. Ajay, ${ }^{1}$ Ashwin Iyer, ${ }^{1}$ \\ Sarah Boswell, ${ }^{2}$ John N. Hutchinson, ${ }^{3}$ Sushrut S. Waikar, ${ }^{1}$ and Vishal S. Vaidya ${ }^{1,2,4}$ \\ ${ }^{1}$ Renal Division, Department of Medicine, Brigham and Women's Hospital (BWH), Boston, Massachusetts, USA. ${ }^{2}$ Harvard \\ Program in Therapeutic Sciences, Harvard Medical School, Boston, Massachusetts, USA. ${ }^{3}$ Department of Biostatistics, \\ ${ }^{4}$ Department of Environmental Health, Harvard T. H. Chan School of Public Health, Boston, Massachusetts, USA.
}

Secreted modular calcium-binding protein 2 (SMOC2) belongs to the secreted protein acidic and rich in cysteine (SPARC) family of matricellular proteins whose members are known to modulate cell-matrix interactions. We report that SMOC2 is upregulated in the kidney tubular epithelial cells of mice and humans following fibrosis. Using genetically manipulated mice with SMOC2 overexpression or knockdown, we show that SMOC2 is critically involved in the progression of kidney fibrosis. Mechanistically, we found that SMOC2 activates a fibroblast-to-myofibroblast transition (FMT) to stimulate stress fiber formation, proliferation, migration, and extracellular matrix production. Furthermore, we demonstrate that targeting SMOC2 by siRNA results in attenuation of TCF $\beta 1$-mediated FMT in vitro and an amelioration of kidney fibrosis in mice. These findings implicate that SMOC2 is a key signaling molecule in the pathological secretome of a damaged kidney and targeting SMOC2 offers a therapeutic strategy for inhibiting FMT-mediated kidney fibrosis - an unmet medical need.

\section{Introduction}

Fibrosis is an aberrant repair response to chronic tissue injury (1). The fairly conserved mechanism of repair makes fibrosis a common end-feature of nearly all chronic inflammatory organ diseases, contributing to the morbidity and mortality of approximately half of the industrialized world (1). The kidney is known for its high susceptibility to injury related, in part, to its elevated concentrations of filtered toxins and predisposition to ischemia, as well as sepsis, rendering it particularly susceptible to fibrosis (2). Kidney fibrosis is also the common pathophysiological phenomenon of a majority of progressive chronic kidney diseases (CKDs) $(3,4)$. The fibrotic events in the kidney are specifically defined by the excessive deposition of a pathological extracellular matrix (ECM) in the interstitial space between tubules and peritubular capillaries, interfering with their normal exchange of toxins and nutrients (5). Myofibroblasts are widely recognized as the effector cells responsible for fibrosis, since they are considered the dominant ECM-producing cells originating via activation of resident fibroblasts by exposure to profibrotic factors, essentially TGF $\beta 1$ and ECM proteins (6-9). Therefore, inhibiting factors that regulate this self-perpetuating loop of ECM production and myofibroblast activation represents a logical approach to target kidney fibrosis that remains to be an unmet medical need.

Previously, using RNA sequencing, we identified secreted modular calcium-binding protein 2 declared that no conflict of interest exists.

Submitted: August 23, 2016 Accepted: March 16, 2017 Published: April 20, 2017

Reference information: JCI Insight. 2017;2(8):e90299. https:// doi.org/10.1172/jici.insight.90299. (SMOC2) to be among the most highly upregulated genes in the kidneys of mice subjected to folic acid-induced (FA-induced) chronic progressive kidney fibrosis (10). SMOC2 belongs to the secreted protein acidic and rich in cysteine (SPARC) family of matricellular proteins, whose members are known for their secretion into the extracellular space to not only interact with structural matrix proteins but also with cell surface receptors, growth factors, proteases, and other bioactive effectors to modulate cell-matrix interactions and cell function (11). Mechanistically, apart from its role in ECM assembly signaling, SMOC2 has been hypothesized to serve as a target for controlling angiogenesis in tumor growth and myocardial ischemia $(12,13)$. Given that there is no information on the function- 
Table 1. Demographics and clinical characteristics of patients with or without chronic kidney disease (CKD).

\begin{tabular}{|c|c|c|c|c|c|c|c|c|}
\hline Participant & Age & Sex & Race & eGFR $\left(\mathrm{ml} / \mathrm{min} / 1.73 \mathrm{~m}^{2}\right)$ & Stage & Cause of CKD & $\begin{array}{l}\text { SMOC2/U Cr } \\
\text { (ng/mg) }\end{array}$ & $\begin{array}{l}\mathrm{KIM}-1 / \mathrm{U} C \mathrm{r} \\
\text { (ng/mg) }\end{array}$ \\
\hline \multicolumn{9}{|c|}{ Chronic kidney disease } \\
\hline 1 & 73 & M & B & 15 & 4 & Diabetes, Hypertension & 3.98 & 1.24 \\
\hline 2 & 62 & $\mathrm{~F}$ & 0 & 19 & 4 & Chronic Interstitial Nephritis & 0.68 & 10.39 \\
\hline 3 & 35 & $\mathrm{~F}$ & W & 20 & 4 & $\begin{array}{c}\text { Congenital Anomalies of the Kidney and Urinary } \\
\text { Tract }\end{array}$ & 0.08 & 1.52 \\
\hline 6 & 75 & $\mathrm{~F}$ & B & 22 & 4 & Diabetes, Hypertension & 0.12 & 0.55 \\
\hline 7 & 64 & M & W & 25 & 4 & Nephrectomy, recurrent Urinary Tract Infections & 2.75 & 0.80 \\
\hline 8 & 56 & $\mathrm{~F}$ & $\mathrm{~B}$ & 20 & 4 & Hypertension & 3.74 & 0.95 \\
\hline 9 & 64 & $\mathrm{~F}$ & W & 13 & 5 & Diabetes and Phosphate Nephropathy & 2.05 & 3.55 \\
\hline 10 & 39 & M & W & 6 & 5 & Chronic Interstitial Nephritis & 5.87 & 1.39 \\
\hline 14 & 21 & $\mathrm{~F}$ & B & - & - & - & 0.28 & 0.43 \\
\hline 15 & 27 & M & W & - & - & - & 0.92 & 0.19 \\
\hline 16 & 21 & $\mathrm{~F}$ & W & - & - & - & 1.93 & 0.90 \\
\hline 17 & 21 & $\mathrm{~F}$ & B & - & - & - & 0.92 & 0.57 \\
\hline 18 & 29 & $M$ & 0 & - & - & - & 1.38 & 1.34 \\
\hline 19 & 19 & $M$ & B & - & - & - & 0.28 & 0.28 \\
\hline 20 & 20 & $M$ & 0 & - & - & - & 0.07 & 0.24 \\
\hline 21 & 19 & $M$ & W & - & - & - & 0.66 & 0.06 \\
\hline 22 & 36 & $\mathrm{~F}$ & W & - & - & - & 0.82 & 0.66 \\
\hline 23 & 19 & $M$ & 0 & - & - & - & 0.66 & 0.49 \\
\hline 24 & 29 & $\mathrm{~F}$ & 0 & - & - & - & 1.75 & 2.16 \\
\hline
\end{tabular}

$\mathrm{U}$ Cr, Urine Creatinine; B, Black; W, White; O, Other.

al significance of SMOC2 upregulation following kidney damage, the objective of this study was to investigate whether induction of SMOC2 in the kidney regulates initiation and progression of kidney fibrosis and whether genetic or pharmacologic modulation of SMOC2 is capable of preventing fibrosis.

\section{Results}

SMOC2 is highly induced in mice and human kidneys following fibrosis. SMOC2 was significantly $(P<0.05)$ induced in mice when subjected to unilateral ureteral obstruction (UUO) or treated with FA (250 mg/ kg, i.p.) (Figure 1, A-D, and Supplemental Figure 1, A-C; supplemental material available online with this article; https://doi.org/10.1172/jci.insight.90299DS1), two mechanistically distinct mouse models of kidney injury with resulting progressive fibrosis $(10,14-21)$. Costaining of SMOC2 with $\alpha$-smooth muscle actin ( $\alpha$ SMA $)+$ myofibroblasts in the kidneys of mice subjected to UUO or FA at 7 days confirmed the widespread upregulation of SMOC2 throughout the fibrotic kidney, predominantly in the proximal and distal tubular epithelial cells around the areas of fibrosis (Figure 1, A and B). In relation to $\alpha$ SMA (Figure 1A, bottom panel), SMOC2 did not colocalize with myofibroblasts; rather, it was expressed around the myofibroblasts that are the effector cells of fibrosis, complying with the extracellular expression of SMOC2. In both UUO and FA models, SMOC2 expression correlated with progression of fibrosis characterized by the expression of $\alpha \mathrm{SMA}^{+}$myofibroblasts, collagen, and fibronectin (Figure 1, C and D, and Supplemental Figure 1, B and C). Translatability of SMOC2 expression in human disease was confirmed by observing a significant induction of SMOC2 in the tubular epithelial cells of human kidney biopsy sections from patients with pathological fibrosis (Figure 1E and Supplemental Figure 1D). SMOC2 being a secreted 
A

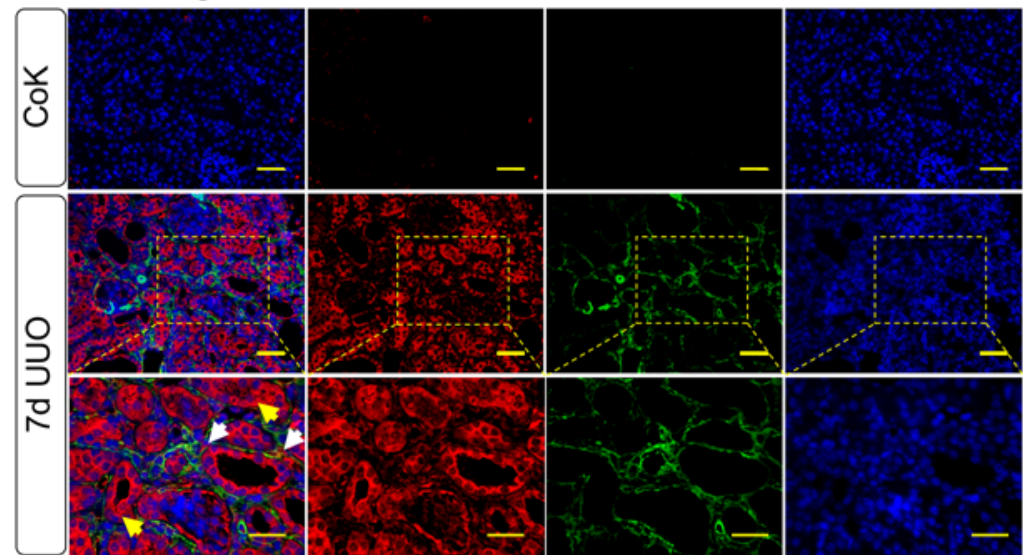

B

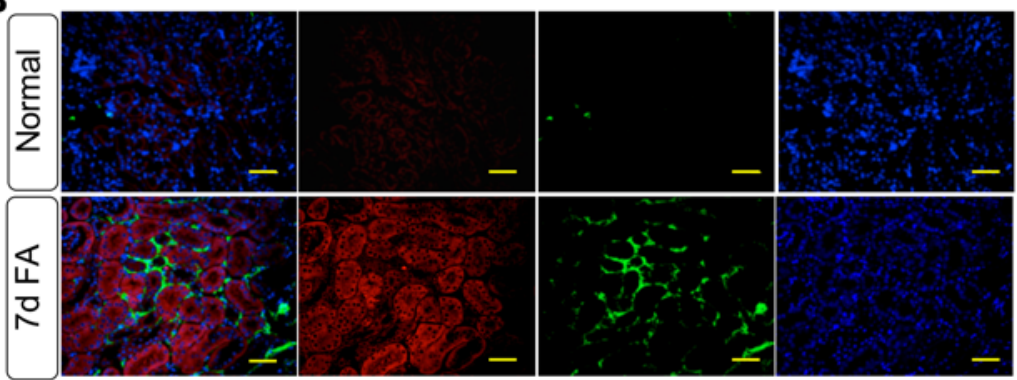

E

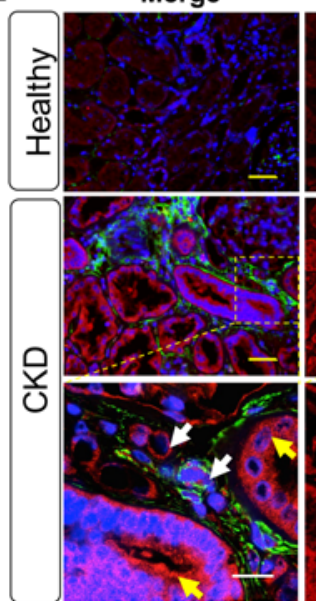

SMOC2

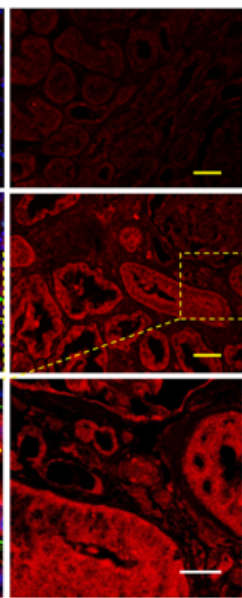

aSMA

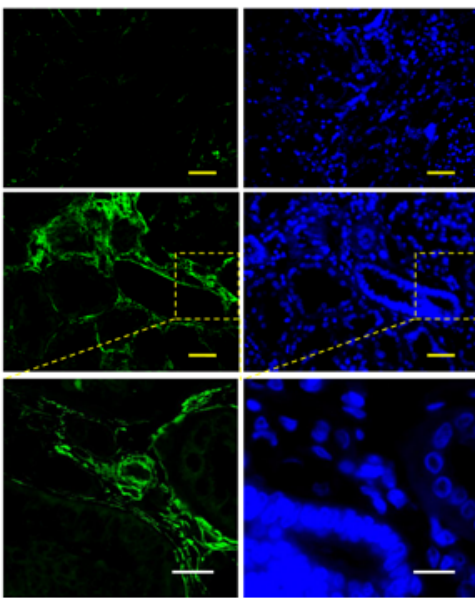

Quantitation of Immunostaining

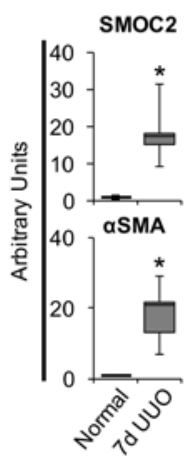

Quantitation of Immunostaining

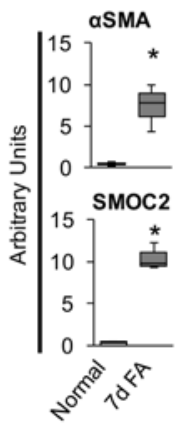

Quantitation of Immunostaining

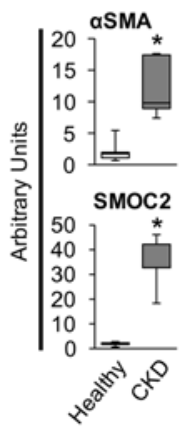

C

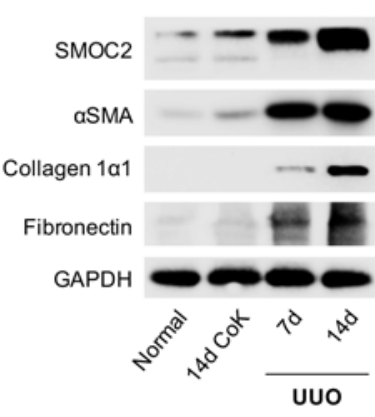

D

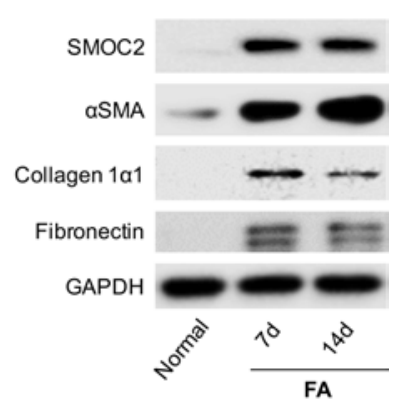

$\mathbf{F}$

Quantitation of Urine

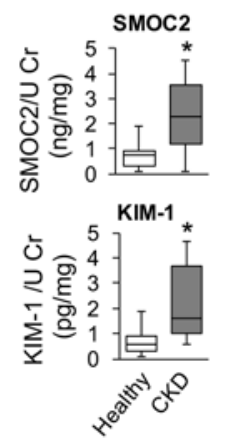

Figure 1. SMOC2 is highly upregulated in mice and humans with kidney fibrosis. Quantitative immunostaining for SMOC2 (red) and $\alpha$ SMA (green) was performed on kidney sections obtained from mice at day 7 following (A) unilateral ureteral obstruction (UUO) or (B) folic acid injection (FA) (n $=5 ; 20 \times$ magnification). Scale bar: $50 \mu \mathrm{m}$. For the UUO model, contralateral kidney (CoK) tissue from day 14 was also included. Bottom panel in A shows magnified images taken at $40 \times$ from the selected regions of the middle row. Scale bar: $30 \mu \mathrm{m}$. Relative quantitation of SMOC2 and $\alpha \mathrm{SMA}$ immunofluorescence, as represented in a box plot, was performed using representative images of 5 visual fields for each tissue analyzed. (C and D) Representative Western blot ( $n=5 /$ condition; Supplemental Figure 1, B and C [UUO and FA, respectively]) of SMOC2, $\alpha$ SMA, collagen 1 $\alpha 1$, and fibronectin expression using kidney samples obtained from mice subjected to 7 and 14 days of UUO or FA. (E) Quantitative immunostaining for SMOC2 (red) and SSMA (green) in human kidneys with pathological fibrosis underlying chronic kidney disease (CKD) $(n=5)$ and nonfibrotic patients ( $n=5 ; 20 \times$ magnification). Yellow scale bars: $50 \mu \mathrm{m}$. Bottom panel shows magnified images taken at $60 \times$ from the selected regions of the middle row. White scale bars: $25 \mu \mathrm{m}$. Relative quantitation of SMOC2 and $\alpha$ SMA immunofluorescence as represented in a box plot was performed using representative images of 5 visual fields for each tissue analyzed. (F) Urinary levels of SMOC2 and kidney injury molecule-1 (KIM-1) normalized to urinary creatinine were measured in patients with CKD $(n=13)$ compared with healthy volunteers $(n=13)$. Box plots describe the median (line within box), upper and lower quartiles (bounds of box), and minimum and maximum values (bars). ${ }^{*} P<0.05$ determined by $t$ test. Yellow arrows, tubules. White arrows, interstitium. 
A
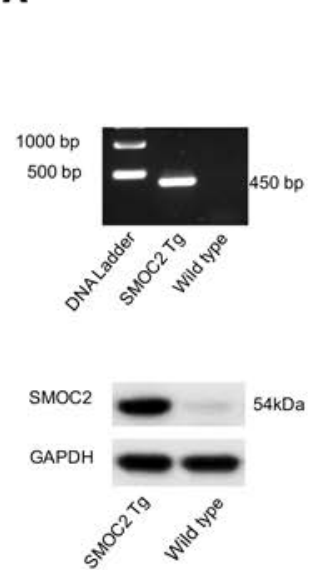

B

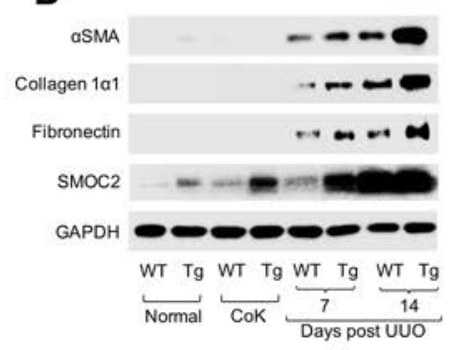

D

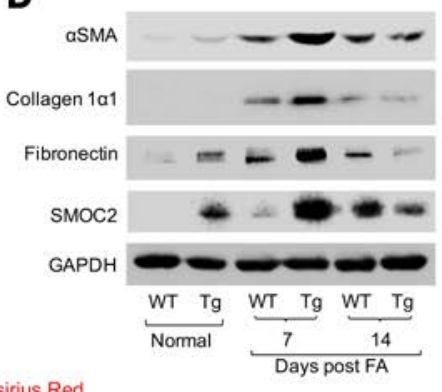

C

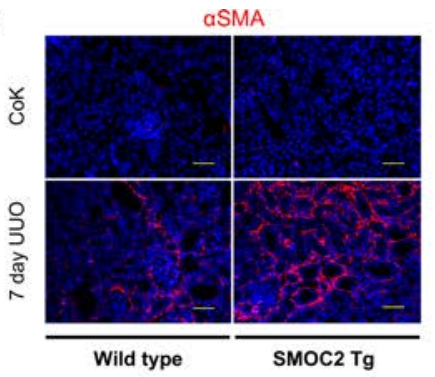

E

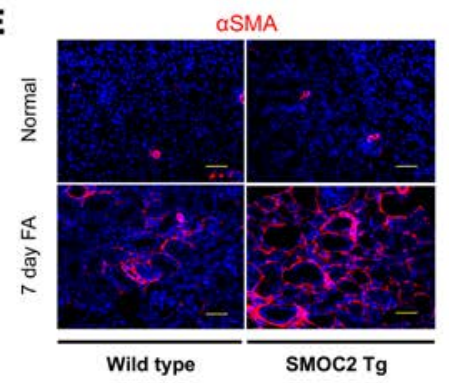

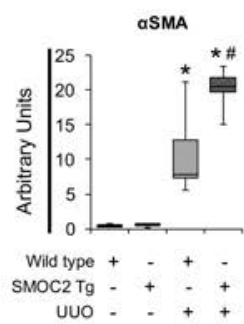

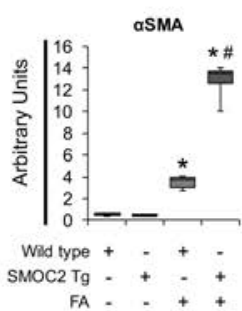

$\mathbf{F}$

Picrosirius Red

Masson's Trichrome
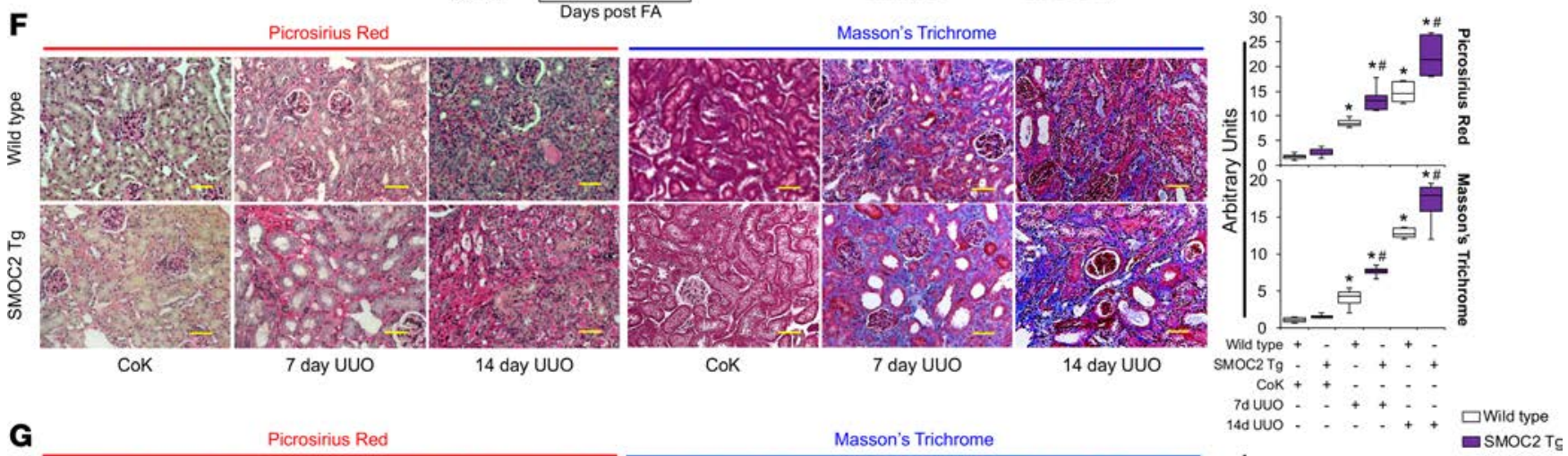

G

Picrosirius Red

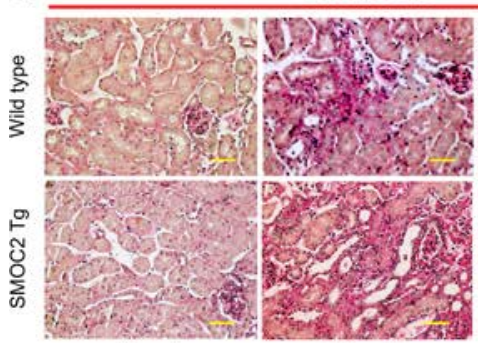

Normal

7 day FA

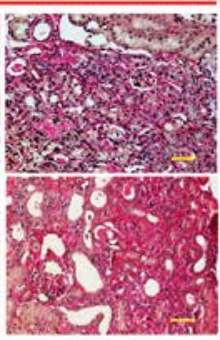

14 day FA

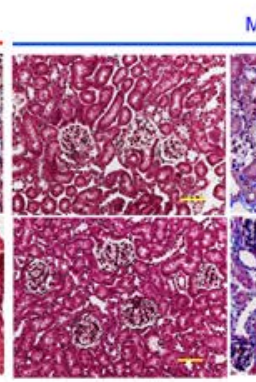

Normal
Masson's Trichrome

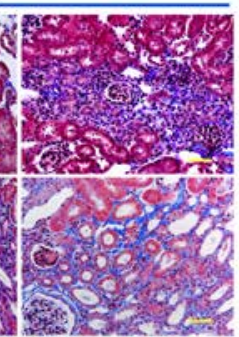

14 day FA

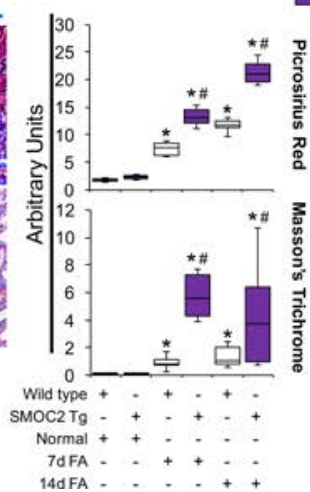

Figure 2. SMOC2-overexpressing mice are more susceptible to kidney fibrosis than WT mice. (A) Confirmation of SMOC2 overexpression in SMOC2 transgenic (SMOC2 Tg) mice by PCR (above, primers specific to recognize Tg insert) and Western blotting (below) (Supplemental Figure 3A). (B) Representative Western blot ( $n=5$ /condition; Supplemental Figure 3B and Supplemental Figure 5B) of $\alpha$ SMA, collagen 1 $\alpha 1$, fibronectin, and SMOC2 expression using kidney samples obtained from SMOC2 Tg and WT mice subjected to 7 and 14 days of unilateral ureteral obstruction (UUO). (C) Representative images of immunofluorescent staining for $\alpha$ SMA in CoK and fibrotic kidneys from WT and SMOC2 Tg mice at day 7 following UUO ( $n=5 /$ condition, 5 visual fields/ tissue). (D) Representative Western blot ( $n=5$ /condition; Supplemental Figure 3C and Supplemental Figure 6B) of $\alpha$ SMA, collagen 1 $\alpha 1$, fibronectin, and SMOC2 expression using kidney samples obtained from SMOC2 Tg and WT mice subjected to 7 and 14 days of folic acid (FA). (E) Representative images of immunofluorescent staining for $\alpha$ SMA of normal and fibrotic kidneys from WT and SMOC2 Tg mice at day 7 following FA ( $n=5 /$ condition, 10 visual fields/ tissue). (F) Representative images of picrosirius red ( $n=5 /$ condition, 10 visual fields/tissue) and Masson's trichrome ( $n=5 /$ condition, 5 visual fields/ tissue) staining of CoK versus 7 and 14 day UUO-treated kidneys. (G) Representative images of picrosirius red and Masson's trichrome staining of normal versus 7 and 14 day FA-treated kidneys ( $n=5 /$ condition, 5 visual fields/tissue). Confocal and light microscopy images are $20 \times$ magnification. Scale bars: $50 \mu \mathrm{M}$. Relative quantifications of images are represented as box plots, which describe the median (line within box), upper and lower quartiles (bounds of box), and minimum and maximum values (bars). ${ }^{*} P<0.05$ (CoK [UUO] or Normal [FA]) and ${ }^{\#} P<0.05$ (WT at respective time point) determined by one-way ANOVA with Tukey post-hoc analysis. 
protein was also significantly elevated $(2.5$ fold, $P<0.05)$ in the urine of patients with CKD (Table $1, n=$ $13)$ as compared with healthy volunteers $(n=13)$. This increase corresponded with the increase in tubular damage biomarker kidney injury molecule-1 (KIM-1, Figure 1F). Consistent with these in vivo findings, SMOC2 expression was also significantly increased in mouse embryonic fibroblasts (NIH3T3) and in primary human proximal tubular epithelial cells (HPTECs) upon treatment with the profibrotic cytokine TGFß1 (10 ng/ml) (Supplemental Figure 2).

SMOC2-overexpressing mice exhibit enhanced kidney fibrosis. SMOC2-overexpressing transgenic mice (SMOC2 Tg) had markedly high SMOC2 levels (Figure 2A and Supplemental Figure 3A) but normal histology of the heart, kidney, liver, lung, spleen, ovary, and testis (Supplemental Figure 4). When subjected to UUO, SMOC2 Tg showed significantly greater fibrosis compared with WT littermates as measured by mRNA (Supplemental Figure 5A) and protein levels (Figure 2B; Supplemental Figure 3B and Supplemental Figure 5B) of fibrotic markers in the kidneys at days 7 and 14 after injury. This correlated with the $\sim 2$-fold greater presence of $\alpha \mathrm{SMA}^{+}$myofibroblasts in the interstitium (Figure 2C). Similarly, SMOC2 Tg mice also demonstrated enhanced fibrosis when treated with FA $(250 \mathrm{mg} / \mathrm{kg}$, i.p.) both at mRNA (Supplemental Figure 6A) and protein (Figure 2D; Supplemental Figure 3C; and Supplemental Figure 6B) levels. Moreover, $\alpha \mathrm{SMA}^{+}$ myofibroblasts in the interstitium (Figure 2E) were significantly elevated in the SMOC2 Tg mice as compared with WT mice following FA treatment. SMOC2 Tg mice also showed consistently higher amounts of pathological tubulointerstitial fibrosis than WT mice, as detected by both picrosirius red and Masson's trichrome staining of the kidneys at days 7 and 14 following UUO (Figure 2F) or FA (Figure 2G).

SMOC2 promotes fibroblast to myofibroblast transition. We performed RNA sequencing in SMOC2 Tg and WT mice kidneys at day 7 following UUO to investigate the mechanisms responsible for increased susceptibility of SMOC2 Tg mice to develop fibrosis (Supplemental Figure 7A). Gene set enrichment analysis (GSEA) for gene ontology (GO) and KEGG terms for cellular components (Supplemental Figure 7, B and C) revealed that genes in the ECM category represented a highly statistically significant difference between SMOC2 Tg and WT mice (Figure 3A). Therefore, we investigated the potential of SMOC2 to transform fibroblasts (human kidney-derived or NIH3T3) (Figure 3, B and C, and Supplemental Figure 8B) into myofibroblasts, which are the major cell type responsible for ECM production. In comparison with an induction of fibroblast-to-myofibroblast transition (FMT) by TGF $\beta 1$, SMOC2 (10 ng/ml, Supplemental Figure $8 \mathrm{~A}$ ) was also capable of inducing FMT as characterized by upregulation of $\alpha \mathrm{SMA}$, collagen $1 \alpha 1$, and fibronectin (Figure 3, B and C; Supplemental Figure 8, B-D). The specificity of SMOC2 to induce FMT was confirmed by preincubating SMOC2 with a SMOC2-specific antibody, which resulted in blocking the SMOC2 signaling effect on fibroblasts (Figure 3D and Supplemental Figure 8, E and F).

SMOC2 has been previously shown to bind keratinocytes through integrin $\beta 1$ (22). To determine if integrin $\beta 1$ binding is the potential mode of action for SMOC2 in fibroblasts, we first treated fibroblasts with an integrin $\beta 1$ antibody prior to SMOC2 treatment. The integrin $\beta 1$ antibody was effective in preventing the induction of FMT markers by SMOC2 (Figure 3D). To confirm their interaction, we immunoprecipitated SMOC2 and then blotted the pull-down for integrin $\beta 1$, and vice versa (Figure $3 \mathrm{E}$ ). The results confirm in a two-way analysis that SMOC2 also binds integrin $\beta 1$ within the fibroblast cell type.

SMOC2 $(10 \mathrm{ng} / \mathrm{ml})$ treatment of quiescent fibroblasts also triggered an early cascade of integrin signaling events for FMT (23), including phosphorylation of focal adhesion kinase (FAK-P) $(24,25)$, myosin light chain (MLC-P) (26), and paxillin (Pax-P) (26), at 45 minutes (Supplemental Figure 8, G and H) with a near double effect at 60 minutes (Figure 3F, Supplemental Figure 8H). As $\alpha$ SMA expression culminates into the assembly of stress fiber, we next validated this structural formation after SMOC2 treatment of fibroblasts (Figure $3 \mathrm{G}$ ). Since RNA sequencing also revealed chemotaxis as a highly significant biological process (Figure 4A and Supplemental Figure 7D) between SMOC2 Tg and WT mice at day 7 following UUO, we investigated chemotactic properties of SMOC2 on fibroblasts by performing a scratch assay and Boyden chamber-based migration assay. Fibroblasts treated with SMOC2 $(10 \mathrm{ng} / \mathrm{ml})$ for 24 hours showed a significantly accelerated closure of the wound (Figure 4B) created by a linear scrape on a monolayer of semiconfluent NIH3T3, which involved a significant repopulation of the wounded area over time (Supplemental Figure 8I). SMOC2 also enhanced migration of fibroblasts by $\sim 50 \%$ (Figure $4 \mathrm{C}$ ). There was also $\sim 9$-fold increase in adhesion following SMOC2 treatment of fibroblasts (Figure 4D). Furthermore, SMOC2 progressively increased the metabolic activity and survival of fibroblasts every 24 hours over the course of 96 hours (Figure 4E and Supplemental Figure 8J). SMOC2 also showed mitogenic properties by stimulating fibroblast proliferation $(P<$ 0.05 ) as assessed by the number of $\mathrm{EdU}^{+}$cells (Figure $4 \mathrm{~F}$ ). In order to validate these effects of recombinant 


\section{A Cellular Components}

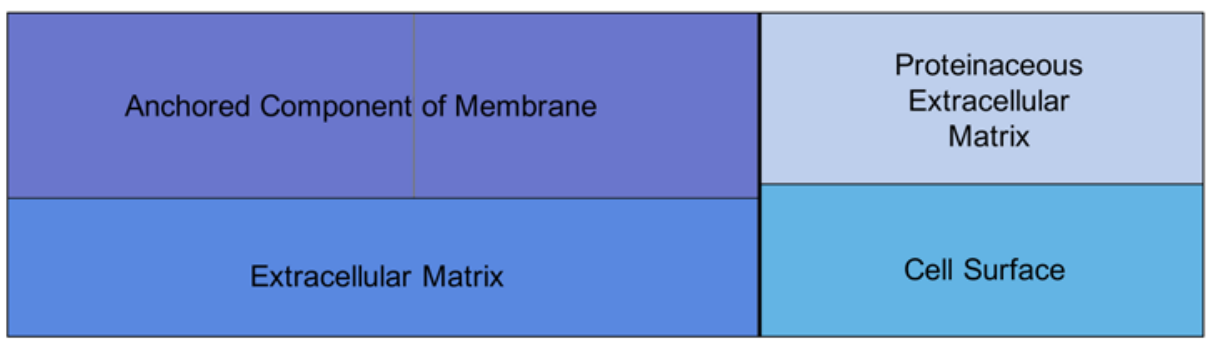

C

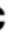 \\ NIH3T3 Fibroblasts} aSMA

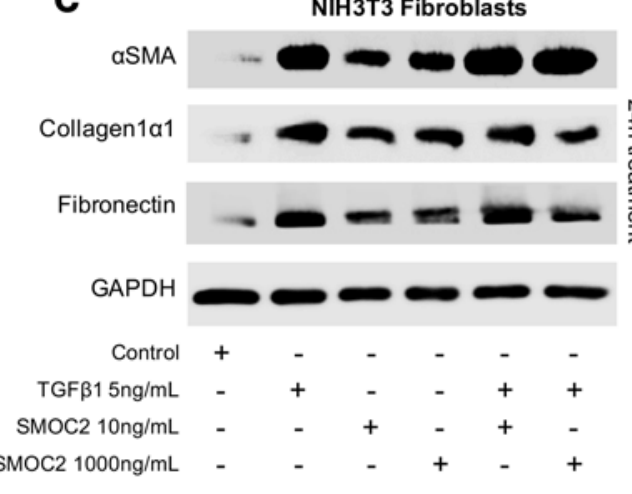

D

D
NIH3T3 Fibroblasts

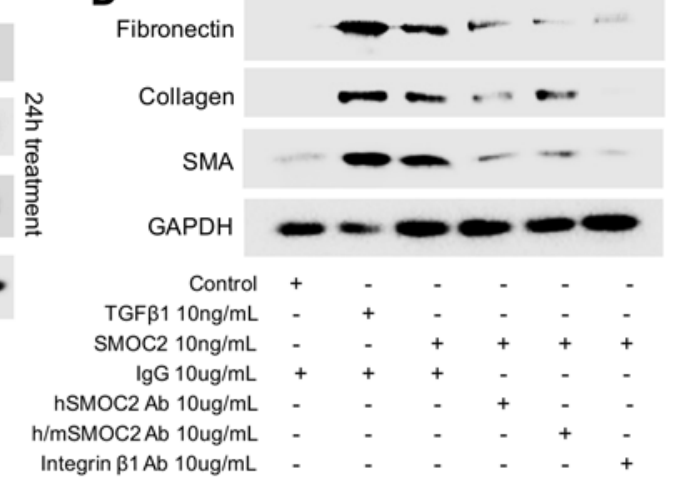

B

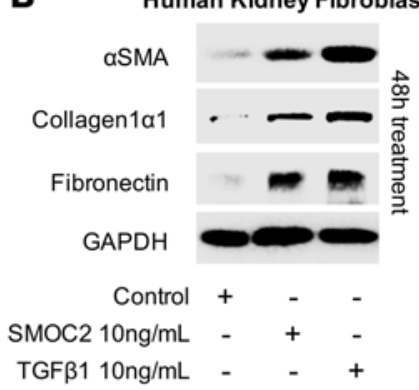

\section{E}
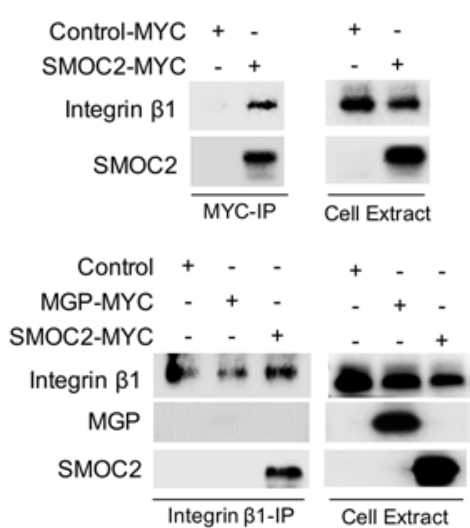
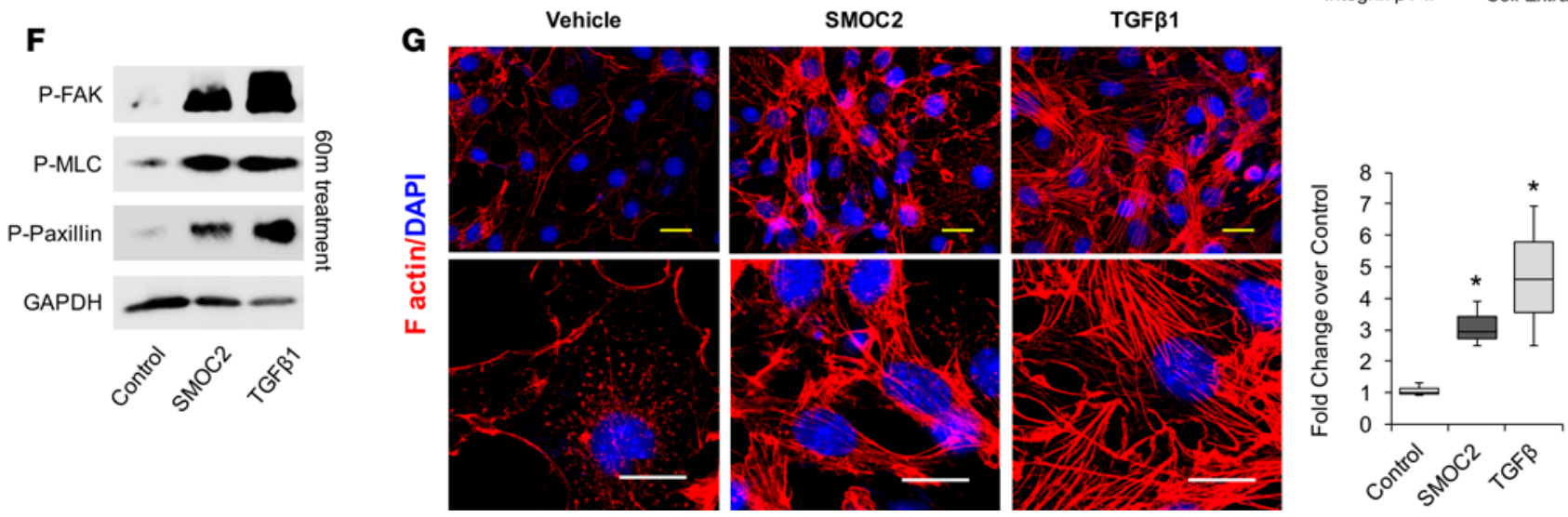

Figure 3. SMOC2 induces a fibroblast-to-myofibroblast transition. (A) RNAseq was performed using kidneys from SMOC2 Tg and WT mice at day 7 following UUO treatment. REVIGO treemap visualizations are shown for enriched gene ontology (GO) categories. Highly similar GO terms for cellular components are grouped and visualized by different colors and sizes of the rectangles using semantic similarity and enrichment $P$ values. Representative Western blots of $\alpha$ SMA collagen 1 1 1, and fibronectin from serum-deprived primary human kidney fibroblasts (B, $n=3 /$ condition; Supplemental Figure 8C) and NIH3T3 fibroblasts (C, $n=$ 3/condition; Supplemental Figure 8D) treated with $10 \mathrm{ng} / \mathrm{ml} \mathrm{SMOC2}$ with/without TCF $\beta 1$. (D) After 1 hour of antibody pretreatment, SMOC2 or TCF $\beta 1$ was treated to serum-deprived NIH3T3 cells for 24 hours and then tested for conventional fibrotic markers, while integrin $\beta 1$ antibody was pretreated with NIH3T3 cells and then treated with SMOC2 ( $n=3$ /condition; Supplemental Figure 8E). (E) NIH3T3 fibroblasts were transfected with SMOC2-MYC, empty vector control, or negative control MCP-MYC and then immunoprecipitated with a MYC (above) or integrin antibody (below). Western blots are representative immunoprecipitation experiments. (F) Representative Western blot for Phospho-Focal Adhesion Kinase (P-FAK) Y925, P-Myosin Light Chain (MLC) Ser19, and P-Paxillin Tyr118 from NIH3T3 cells treated with $10 \mathrm{ng} / \mathrm{ml} \mathrm{SMOC2}$ or $5 \mathrm{ng} / \mathrm{ml} \mathrm{TGF} \beta 1$ for 60 minutes ( $n=5 /$ condition; Supplemental Figure 8H). (C) Phalloidin staining of F-actin after NIH3T3 cells were treated 24 hours with $10 \mathrm{ng} / \mathrm{ml} \mathrm{SMOC2}$ or $5 \mathrm{ng} / \mathrm{ml} \operatorname{TCF} \beta 1(n=3)$. Top row $40 x, 25 \mu \mathrm{M}$. Bottom row 100x, $15 \mu \mathrm{M}$. Box plots describe the median (line within box), upper and lower quartiles (bounds of box), and minimum and maximum values (bars). ${ }^{*} P<0.05$ determined by $t$ test.

SMOC2 on fibroblasts, we also created SMOC2-overexpressing fibroblasts by transfecting NIH3T3 cells with pCMV-SMOC2 and observed similar phenotypic changes (Supplemental Figure 9). Taken together, these results suggest that SMOC2 stimulates FMT signaling with activation of its characteristic features, including metabolic activity, proliferation, migration, and adhesion.

SMOC2-KO mice are protected from kidney fibrosis. In order to investigate the effect of inhibition of SMOC2 on fibrosis progression, we first used a genetic manipulation approach and confirmed that 
A Biological Processes

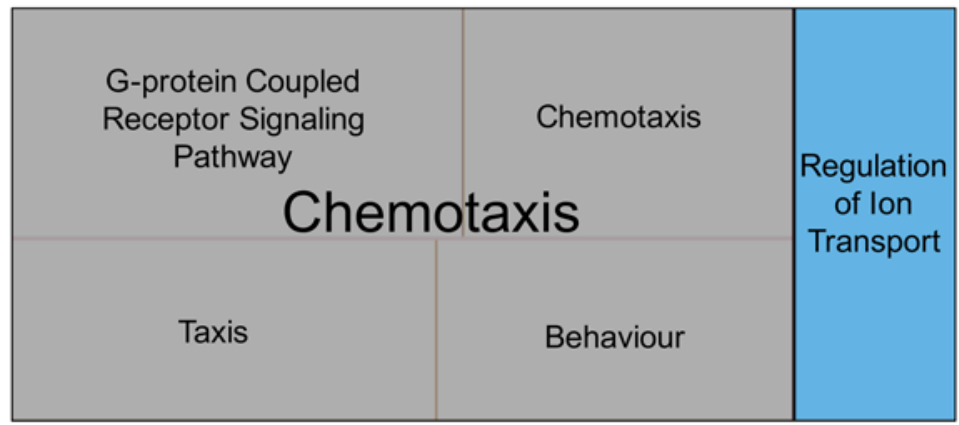

B

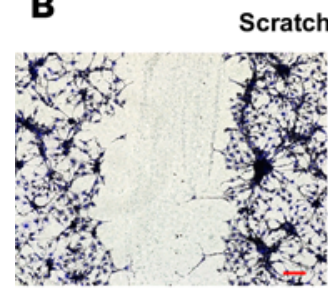

Control
Scratch Assay

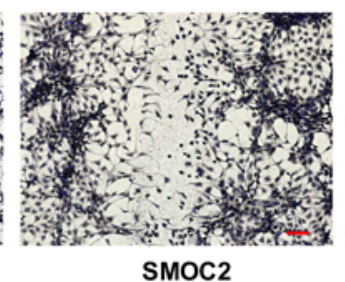

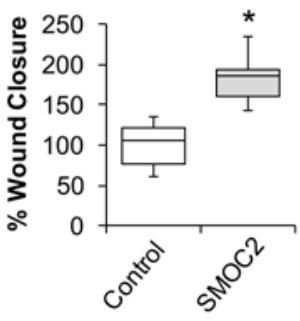

C

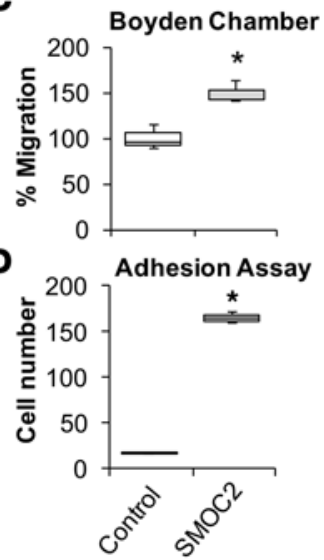

E

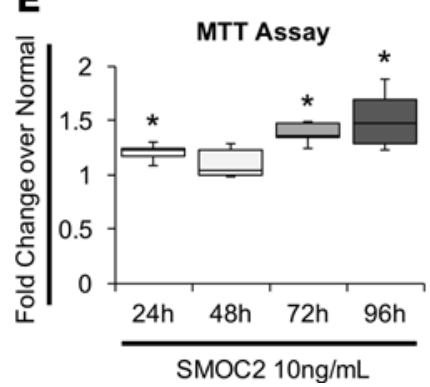

$\mathbf{F}$
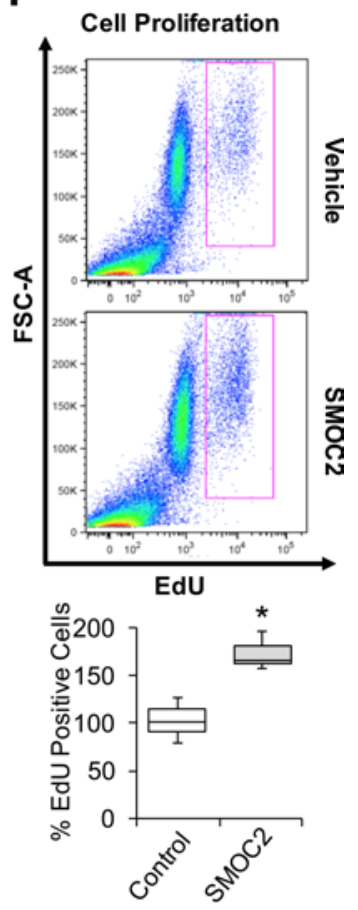

Figure 4. SMOC2 induces the properties of myofibroblast activities. (A) REVIGO treemap visualization for highly similar GO terms describing biological processes significantly different between SMOC2 Tg and WT mice at day 7 following UUO treatment. (B) Scratch assay performed on NIH3T3 cells treated 24 hours with $10 \mathrm{ng} / \mathrm{ml} \mathrm{SMOC2}$. Healing percentage represented in box plot ( $n=5,3$ visual fields/condition; $10 \times$ magnification, scale bar: $50 \mu \mathrm{M})$. (C) Boyden chamber assay performed on NIH3T3 cells treated 24 hours with $10 \mathrm{ng} / \mathrm{ml} \mathrm{SMOC2}$. (D) NIH3T3 cells were treated 24 hours with/without $10 \mathrm{ng} / \mathrm{ml}$ SMOC2, and then trypsinized and reseeded. After 1 hour, unattached cells were washed and cell numbers were quantified for adherence ( $n=3)$. (E) Metabolic activity of control and $10 \mathrm{ng} / \mathrm{ml}$ SMOC2-treated NIH3T3 cells were measured over time by MTT assay $(n=5)$. (F) NIH3T3 fibroblasts were treated 24 hours with/without $10 \mathrm{ng} / \mathrm{ml} \mathrm{SMOC2}$, and cell proliferation was assessed by EdU labeling and FACS $(n=5)$. Box plots describe the median (line within box), upper and lower quartiles (bounds of box), and minimum and maximum values (bars). ${ }^{*} P<0.05$ determined by $t$ test.

SMOC2-KO mice (Figure 5A) were histologically normal (Supplemental Figure 10). When the SMOC2KO mice were subjected to FA (Figure 5) and UUO (Figure 6), there was a marked attenuation of SMOC2 and fibrotic markers at day 7 as compared with WT mice (Figure 5B; Supplemental Figure 11; Figure 6A; Supplemental Figure 12). This was confirmed by a significant decrease in $\alpha \mathrm{SMA}^{+}$cells in the interstitium (Figure 5C and Figure 6B), along with significant reduction in deposition and accumulation of ECM visualized by Masson's trichrome staining (Figure 5D and Figure 6C).

Targeting SMOC2 using RNA interference protects against fibrosis development. Next, we used a pharmacological inhibition approach to inhibit SMOC2 by synthesizing small interfering RNAs (siRNAs). We tested the efficacy of 4 siRNAs and found one (target sequence: UCUGAACUCUGAAUUUAA) that resulted in $~ 90 \%$ silencing in vitro (SMOC2 siRNA \#16 in Supplemental Figure 13). NIH3T3 cells transfected with SMOC2 siRNA resulted in significant attenuation of TGF $\beta 1$-mediated FMT and signaling as measured by a significant decrease in SMOC2, $\alpha$ SMA, collagen 1 $\alpha 1$, and fibronectin expression (Figure 7A; Supplemental Figure 14). Using the same SMOC2 siRNA sequence, we then synthesized endotoxin-free, chemically modified SMOC2 siRNA that is resistant to degradation in vivo and localizes in the kidneys (27) (Supplemental Figure 15). SMOC2 siRNA, when injected into mice i.v., also resulted in $\sim 50 \%$ reduction in kidney SMOC2 protein expression following FA administration (Figure 7B, Supplemental Figure 16), thereby establishing proof of delivery. More importantly, a significant amelioration of kidney fibrosis was observed in mice treated with SMOC2 siRNA as compared with scrambled siRNA at day 7 following FA treatment (Figure 7B, Supplemental Figure 16). Myofibroblast transformation and collagen accumulation as assessed by $\alpha$ SMA staining and Masson's trichrome staining, respectively, was significantly less in FA-injected mice treated with SMOC2 siRNA (Figure 7, C and D) compared with scrambled siRNA-treated (ssiRNA-treated) mice. 
A
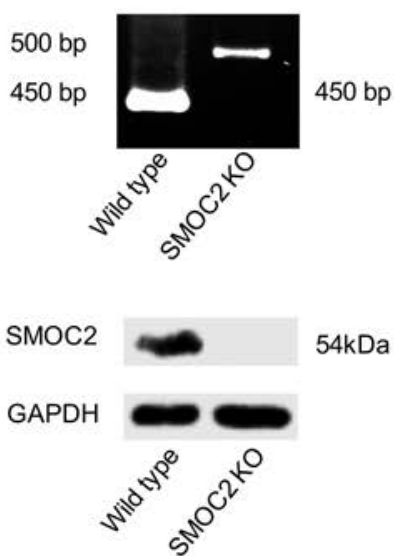

B

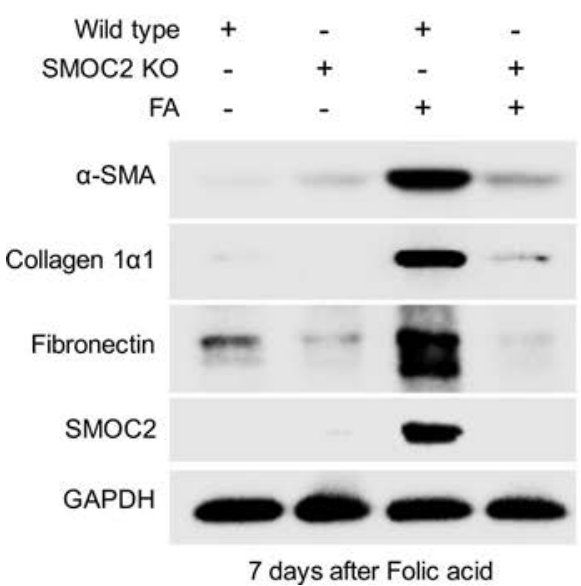

C

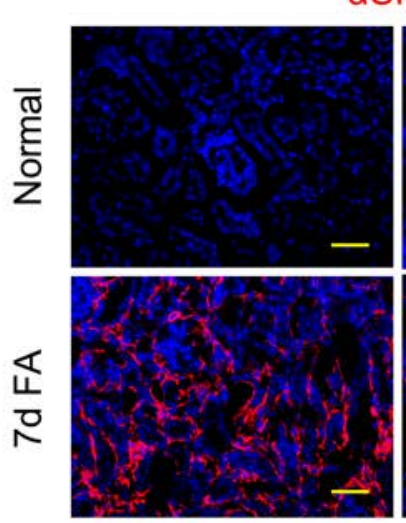

Wild type

D

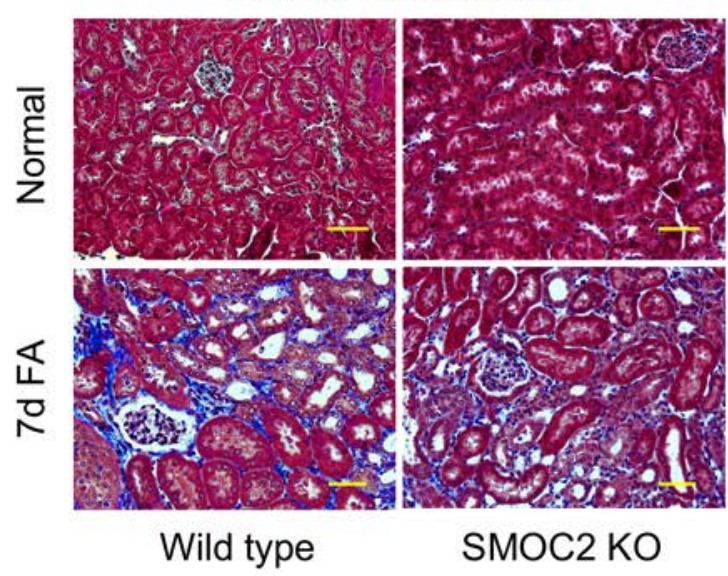

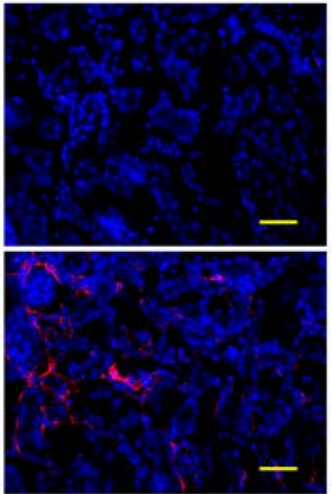

SMOC2 KO

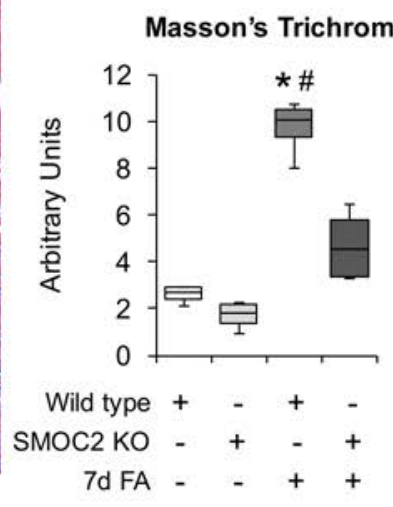

Figure 5. Genetic inhibition of SMOC2 limits folic acid-induced kidney fibrosis in mice. (A) Confirmation of SMOC2 deletion in SMOC2-KO mice by PCR

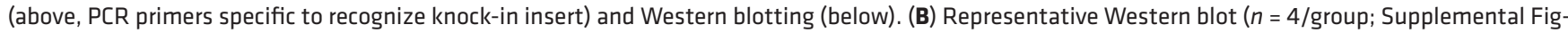
ure 11) of $\alpha$ SMA, collagen 1 11 , fibronectin, and SMOC2 expression using kidney samples obtained at day 7 from SMOC2-KO and WT mice subjected to folic acid (FA) treatment. (C) Immunofluorescent $\alpha$ SMA staining of KO and WT kidneys at day 7 following with/out FA treatment ( $n=4 /$ group). (D) Masson's trichrome staining of normal and FA-treated kidneys obtained at day 7 from KO and WT mice. Confocal and light microscopy images are 20x magnification Scale bars: $50 \mu \mathrm{M}$. Quantification of images is represented as box plots ( $n=4 /$ condition, 10 visual fields/mice), which describe the median (line within box), upper and lower quartiles (bounds of box), and minimum and maximum values (bars). ${ }^{*} P<0.05$ (WT normal) and ${ }^{\#} P<0.05$ (WT at respective treatment) determined by one-way ANOVA with Tukey post-hoc analysis.

\section{Discussion}

The stroma's composition and organization of ECM proteins are integral signaling features that dictate the cause and effect of persistent fibroblast activation, underlying pathological fibrosis (28) and, as a result, the ongoing loss of normal tissue structure. The present study systematically supports the notion that the matricellular factor SMOC2 is minimal under normal conditions but upregulated upon kidney injury to eventually partake in the deleterious response of fibrosis. We provide evidence to suggest (i) SMOC2 expression is significantly induced in the kidneys of mice and humans following fibrosis, irrespective of the mechanism of initiation of fibrosis; (ii) SMOC2 is critically involved in kidney fibrosis progression because transgenic mice overexpressing SMOC2 exhibit significantly enhanced tubulointerstitial fibrosis, whereas SMOC2-KO mice are protected from kidney fibrosis development; (iii) Inhibition of SMOC2 in vitro and in vivo using siRNA protects from fibrosis progression, suggesting SMOC2 as a potential therapeutic target for kidney fibrosis; and (iv) mechanistically, SMOC2 activates matrix assembly signaling in the fibroblasts to stimulate stress fiber formation, proliferation, migration, and ECM production - features typical of transitioning into myofibroblasts, which are the effector cells in fibrosis.

FMT is a quintessential phase in the development of fibrosis because of the central role myofibroblasts have in the production of collagen and fibronectin. We hypothesized and provide experimental 
A

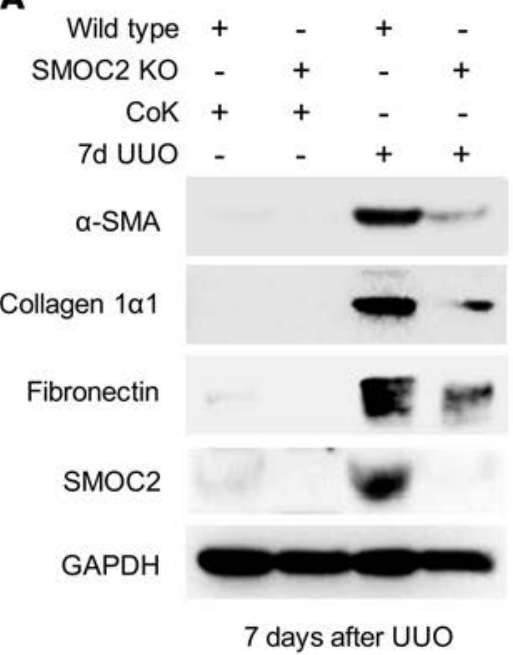

B

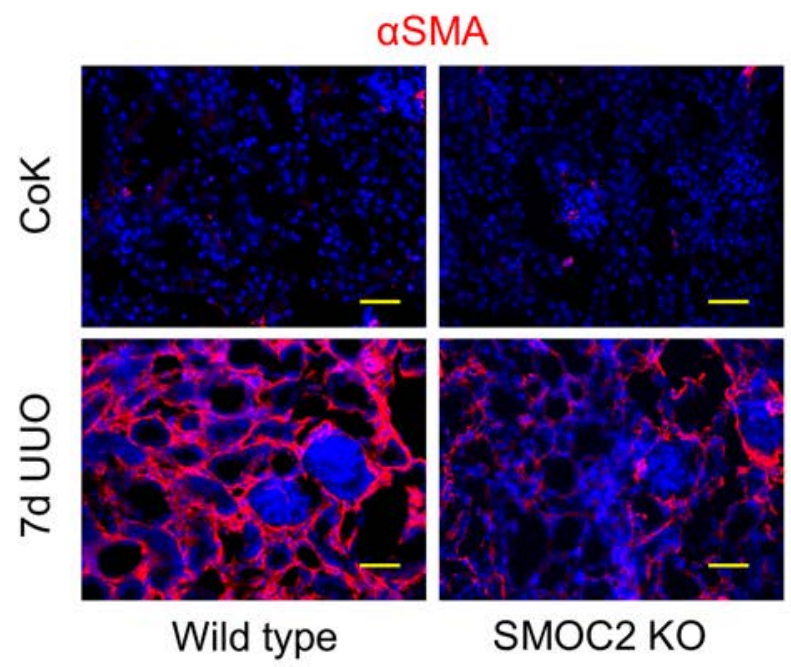

aSMA

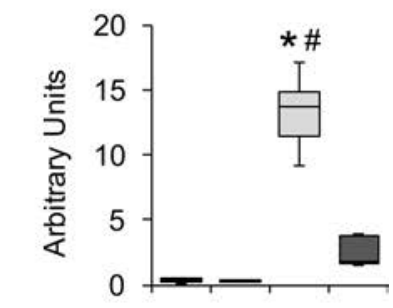

Wild type + - + SMOC2 $\mathrm{KO}-++\quad+$

$\mathrm{CoK}++-$ 7d UUO - - + +
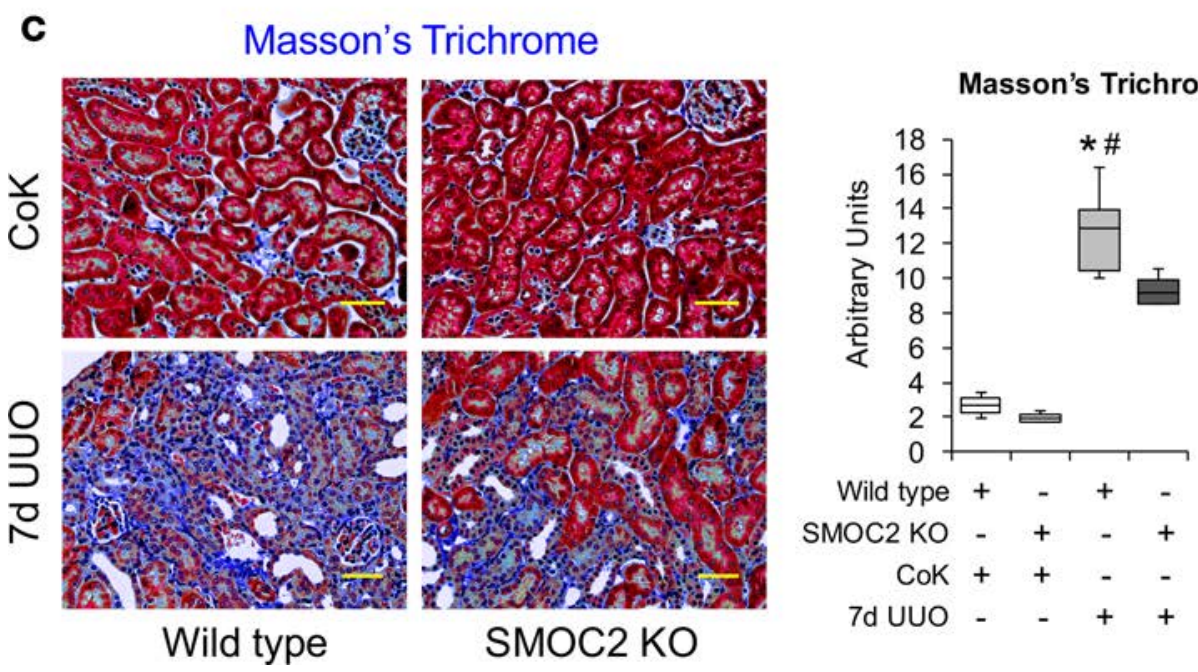

Figure 6. Genetic inhibition of SMOC2 limits UUO-induced kidney fibrosis in mice. (A) Representative Western blot ( $n=5 /$ group; Supplemental Figure 12) of $\alpha$ SMA, collagen $1 \alpha 1$, fibronectin, and SMOC2 expression using kidney samples obtained at day 7 from SMOC2-KO and WT mice subjected to UUO. (B) Representative images ( $n=3 /$ group; 5 visual fields for each tissue analyzed) of immunofluorescent $\alpha$ SMA staining of KO and WT kidneys from normal mice and day 7 UUO mice. Relative quantitation is represented in a box plot as arbitrary units. (C) Masson's trichrome staining of normal and 7-day UUO kidneys from WT and KO mice. Images of Masson's trichrome staining are representative of 5-10 visual fields for each tissue analyzed. Quantification is represented in a box plot as arbitrary units (mice $n=5-6,5-10$ visual fields/mice). Confocal images are $20 \times$ magnification; scale bar: $50 \mu \mathrm{M}$. Light micros-

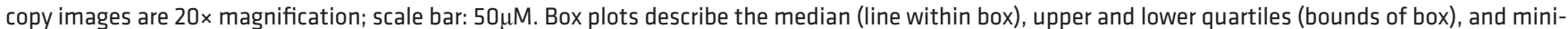
mum and maximum values (bars). ${ }^{*} P<0.05$ (WT CoK) and ${ }^{\#} P<0.05$ (WT at respective UUO) determined by one-way ANOVA with Tukey post-hoc analysis.

verification that SMOC2 is a key signaling molecule in the pathological secretome of a damaged kidney, whose continual presence leads to fibrosis. As the TGF $\beta$ pathway is a hallmark pathway for FMT, we initially found that it was capable of increasing SMOC2 in vitro in fibroblasts and epithelial cells, and we also discovered that SMOC2 ablation significantly attenuated TGF $\beta$-induced FMT, making SMOC2 a potential pathological contributor to fibrosis downstream of TGF $\beta$. Although SMOC2 has not been previously associated with any form of fibrosis, its family member SPARC has been studied extensively in multiple types of fibrosis. The level of SPARC expression was found to be increased in patients with pulmonary, kidney, hepatic, and dermal fibrosis (29). Furthermore, SPARC-null mice had significantly less collagen deposition in the skin, heart, lungs, and kidney upon induction of fibrotic stimuli (29). While both SPARC and SMOC2 promote fibrosis, they most probably differ in their mechanism of action to mediate the interaction between the ECM and cell. SPARC is known for its binding to collagen and postsynthetic processing and assembly of collagen into bundling structures 
A
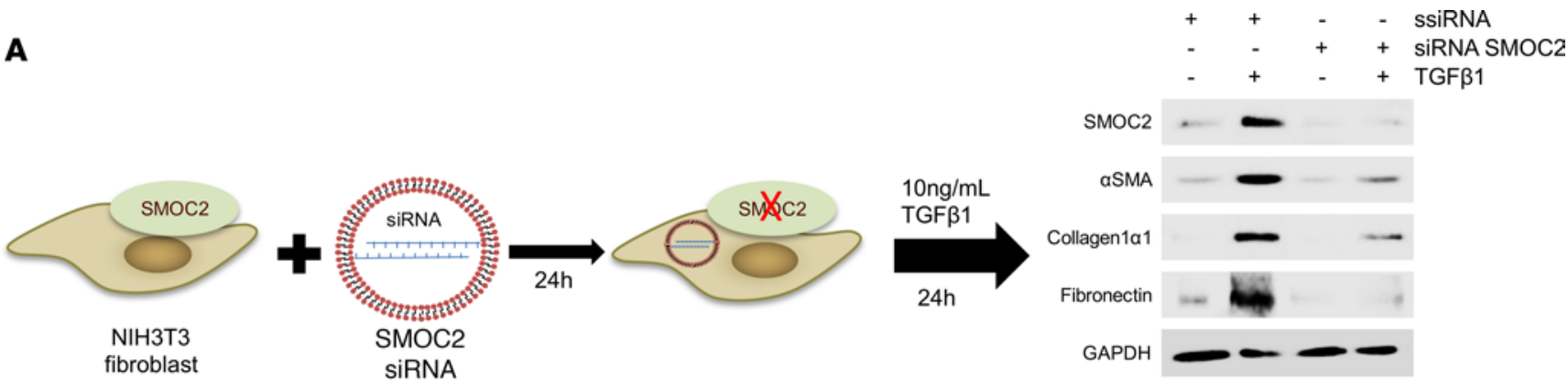

B

Scrambled or SMOC2 siRNA 30 $\mu \mathrm{g} / 200 \mu \mathrm{l}$, intravenous
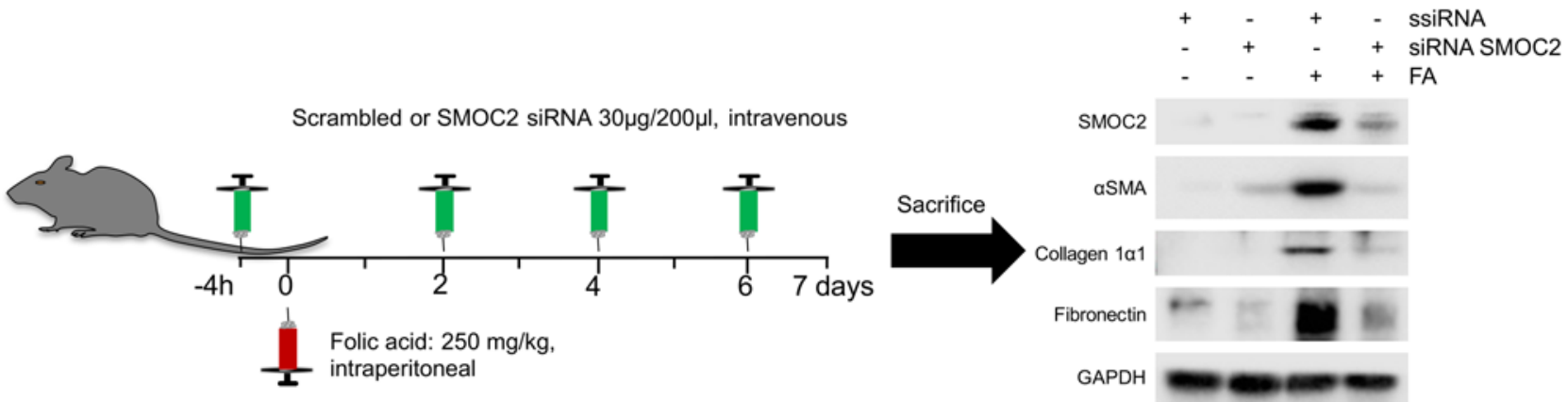

C

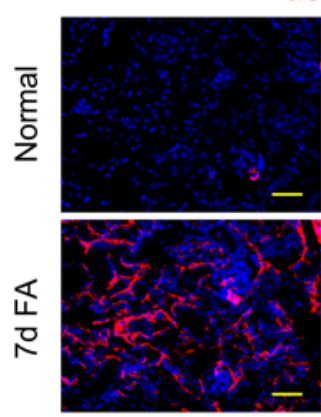

ssiRNA
aSMA

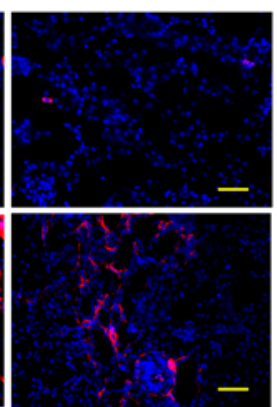

SMOC2 siRNA

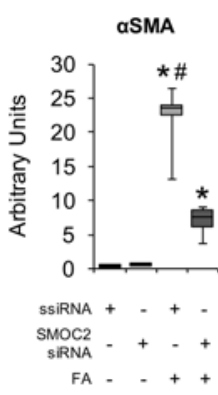

$\mathrm{FA} \cdot++$
D

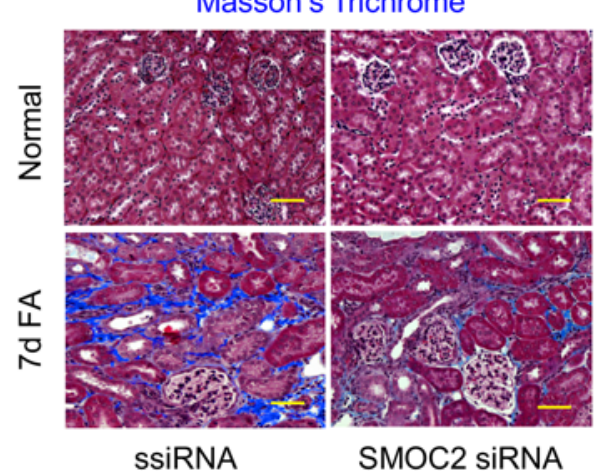

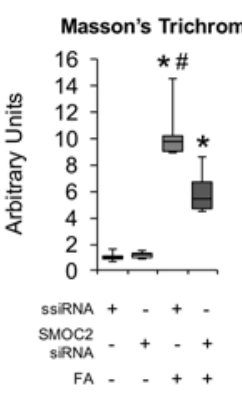

Figure 7. Silencing SMOC2 reduces TGF $\beta 1$-induced fibrotic markers in vitro and folic acid-induced kidney fibrosis in mice. (A) Scheme of the experimental procedure for SMOC2 siRNA-transfected NIH3T3 cells. After 24 hours of treatment with SMOC2 siRNA or scrambled siRNA (ssiRNA), NIH3T3 fibroblasts were either treated with/without TGF $\beta 1$ for 24 hours. Representative Western blot ( $n=3 /$ condition; Supplemental Figure 14) was performed for SMOC2, $\alpha$ SMA, collagen 1 $\alpha 1$, and fibronectin expression. (B) Scheme of the experimental procedure for SMOC2 siRNA- or SsiRNA-injected C57BL/6 mice treated with/out folic acid (FA). Mice were injected i.v. with $30 \mu \mathrm{g} / 200 \mu \mathrm{l}$ of SMOC2 siRNA or ssiRNA 4 hours before and 2, 4, and 6 days after an i.p. injection of $250 \mathrm{mg} / \mathrm{kg}$ of FA. Representative Western blot ( $n=5 /$ group; Supplemental Figure 16) was performed for SMOC2, $\alpha$ SMA, collagen 1 $\alpha 1$, and fibronectin. (C) Immunofluorescent $\alpha$ SMA staining of kidneys obtained from mice at day 7 following with/out FA either treated with ssiRNA or SMOC2 siRNA $(n=5)$. (D) Masson's trichrome staining of normal and FA-treated kidneys obtained at day 7 following ssiRNA or SMOC2 siRNA administration. Confocal and light microscopy images are 20x magnification. Scale bars: $50 \mu \mathrm{M}$. Quantification of images is represented as a box plot ( $n=5 /$ condition, 10 visual fields/mice), which describe the median (line within box), upper and lower quartiles (bounds of box), and minimum and maximum values (bars). ${ }^{*} P$ $<0.05$ (ssiRNA + vehicle) and ${ }^{\#} P<0.05$ (ssiRNA respective treatment) determined by one-way ANOVA with Tukey post-hoc analysis.
$(30,31)$; however, the structure of SMOC2 lacks collagen binding sites such as SPARC to mediate the same effects. This would imply a different mechanism of action, whereby each SPARC member has its respective role in fibrosis development.

SMOC2 is expressed in the heart, muscle, spleen, and ovaries (22), and its expression pattern during development suggests that it may mediate intercellular signaling and cell type-specific differentiation during gonad and reproductive tract development (32). Although we similarly detected SMOC2 expression in normal kidneys (22), overexpression of SMOC2 in mice in the absence of damage did not dispose the mouse kidney to a spontaneous fibrosis; however, the overexpression of SMOC2 in the transgenic mice accelerated a fibrotic response over the WT only after injury. Mechanistically, SMOC2 has been shown 
to act on diverse cell types such as stimulating migration and adhesion of keratinocytes through integrin ( $\alpha \mathrm{v} \beta 1$ and $\alpha \mathrm{v} \beta 6$ ) interaction (22); on endothelial cells where SMOC2 potentiates the responses of VEGFand FGF-induced mitogenesis and angiogenesis (33); and on fibroblasts where SMOC2 regulates cell cycle progression via integrin-linked kinase activity and cyclinD1 expression (34). Matricellular proteins are implicated in regulating the interactions between ECM components and cell surface integrins (35). Integrin $\alpha \beta$ heterodimers translate changes in ECM signals into the fibroblast to undergo FMT $(6,36)$. This mechanosensitive pathway that underlies FMT can be summarized in a three-tier cascade process using the following associated markers (23): FAK-P, MLC-P, and Pax-P. Although we confirm this pathway activation by SMOC2, we provide upstream evidence that integrin $\beta 1$ is part of the initiating process of SMOC2 signaling in fibroblasts. This study does not address whether the effects of SMOC2 are local or systemic because of the use of global SMOC2 transgenic, as well as global SMOC2-KO mouse models. The SMOC2 siRNA delivery was also systemic and not kidney specific. Additional mechanistic studies need to be conducted using conditional SMOC2 mouse models or kidney-specific drug delivery systems to investigate the local vs. systemic effects of SMOC2 on fibrosis.

In summary, we have uncovered a pathway in the pathogenesis of kidney fibrosis initiated by the matricellular protein SMOC2. We show that SMOC2 is critical for the development of kidney fibrosis by stimulating matrix assembly signaling, chemotaxis, and myofibroblast transitioning. We also provide compelling evidence to suggest that silencing SMOC2 to limit fibrosis holds potential as a therapeutic approach to a disease process that has yet to yield promising results.

\section{Methods}

\section{Human studies}

Urine samples from patients with CKD were obtained from the BWH ambulatory nephrology clinic. For this study, we included patients with stage 4 or 5 CKD (estimated glomerular filtration rate [eGFR] $<30$ $\left.\mathrm{ml} / \mathrm{min} / 1.73 \mathrm{~m}^{2}\right)$. Patients were excluded if they had a recent hospitalization or episode of $\mathrm{AKI}(>50 \%$ rise in serum creatinine over a 1-week period) within 3 months or if they reported or suspected urinary tract infection within the past 3 weeks. Urine samples from healthy volunteers were obtained from the PhenoGenetics Project, a study of the impact of genetic variation in healthy individuals. Participants 19-75 years of age were recruited from the Boston area through advertisements in local media and flyers. The inclusion criterion was a willingness to provide $120 \mathrm{ml}$ of blood four times per year for five years. Exclusion criteria were the presence of self-reported inflammatory diseases (e.g., asthma or psoriasis), autoimmune diseases (e.g., lupus of multiple sclerosis), chronic metabolic diseases (e.g., thyroid disease or diabetes), or chronic infections (e.g., Hepatitis B or C; HIV). Urine was collected and centrifuged at 3,200 $g$ for 5 minutes, and the supernatants were collected and stored at $-80^{\circ} \mathrm{C}$ within 4 hours of collection. Deidentified human kidney tissue samples from patients with or without severe kidney fibrosis $(n=10)$ were obtained from the Department of Pathology at BWH.

\section{Animal studies}

Genetic mouse models. SMOC2 Overexpressing Transgenic (Tg[Smoc2-EGFP]HY194Gsat/Mmucd) (SMOC2 Tg) mice were purchased by the University of California, Davis, and generated using a modified BAC, containing an inserted EGFP upstream of targeted SMOC2 gene, that was injected into pronuclei of FVB/N fertilized oocytes. Hemizygous progeny was mated to IcrTac:ICR mice (from UCD) each generation thereafter for maintenance of SMOC2 overexpressing transgenic mice. Smoc2 ${ }^{\operatorname{tm} 1.1}$ (KOMP)Vlcg was generated by the Knockout Mouse Phenotyping Program (KOMP ${ }^{2}$ ) at The Jackson Laboratory using embryonic stem (ES) cells provided by the International Knockout Mouse Consortium. The ZEN-UB1 Velocigene cassette was inserted into the gene, replacing all coding exons and intervening sequences. The construct was introduced into C57BL/6N-derived VGB6 ES cells, and correctly targeted ES cells were injected into B6 (17)-Tyrc-2J/J (stock no. 58) blastocysts. The resulting chimeric males were bred to C57BL/6J females and then to B6N.Cg-Tg(Sox2-cre)1Amc/J (stock no. 014094) to remove neo cassette. Resulting offspring were crossed to remove the cre-expressing transgene. Genotyping was performed using appropriate primers (Supplemental Table 1). Genetic mouse models were compared with their respective WT littermates. All C57BL/6J mice used for experimentation were purchased from Charles River Laboratories. 
Experimental models of fibrosis. Mouse models of kidney fibrosis were used as previously described in detail by our group (14). The following models are briefly described:

FA model. Under the same housing/diet conditions, male SMOC2 Tg with their matched strain control (FVB/N and IcrTac:ICR) (25-29 g), male SMOC2-KO mice with their strain-matched control (C57BL/6) (21-24 g), and male BALB/c mice (25-29 g) aged 8-12 weeks received a single i.p. injection of $250 \mathrm{mg} / \mathrm{kg}$ FA dissolved in a $0.3 \mathrm{M}$ sodium bicarbonate solution (14). Mice were euthanized at 7 and 14 days following administration. Euthanasia was performed under isoflurane anesthesia.

Unilateral ureter obstruction (UUO) model. Female SMOC2 Tg mice with their matched strain control (FVB/N and IcrTac:ICR) (25-29 g), and male BALB/c mice (25-29 g) aged 8-12 weeks were anesthetized $(50 \mathrm{mg} / \mathrm{kg}$ pentobarbital sodium, i.p.), and their left kidney was exposed by flank incision. The ureter was ligated at two points proximal to the kidney with 6-0 sutures (Ethicon; Perma-hand Silk/Black Braided K882). Sham mice had kidney exposed, but their ureter was not tied. Contralateral kidney (CoK) tissue was isolated 14 days after UUO treatment of SMOC2 Tg and WT. Mice received fluid lost replacement $(1 \mathrm{ml}$ normal saline, heated at $37^{\circ} \mathrm{C}$, s.c.) immediately after surgery. The animals were sacrificed at 7 and 14 days following surgery. Euthanasia was performed under isoflurane anesthesia.

siRNA administration. Male C57BL/6 mice (21-24 g) aged 8 weeks received siRNA SMOC2 (30 $\mu \mathrm{g} / 200$ $\mu 1)$ or control scrambled siRNA $(30 \mu \mathrm{g} / 200 \mu \mathrm{l})$ in RNAse-free PBS carriage medium by i.v. injection at $-4 \mathrm{~h}$, $+2 \mathrm{~d},+4 \mathrm{~d}$, and $+6 \mathrm{~d}$ from FA/vehicle treatment.

\section{Pathology and immunostainings}

Whole body pathology. Whole mouse necropsy was performed on male and female mice ( $n=6 /$ each) of all 4 groups (SMOC2-KO, SMOC2-Tg, and their respective littermate controls) to investigate pathological differences between the groups. Organs were formalin-fixed, dehydrated in $70 \% \mathrm{EtOH}$, paraffin-embedded, and H\&E stained. The Dana-Farber/Harvard Cancer Center pathology core led by Peter Howley provided a detailed certified report for histological analysis of all organs.

Histology and staining. For histologic evaluation, kidney tissues were perfused with cold PBS before harvesting. Samples for immunofluorescence were fixed in $4 \%$ paraformaldehyde at $4^{\circ} \mathrm{C}$ for 24 hours, then washed in $30 \%$ sucrose solution overnight prior to cryopreservation in Tissue-Tek O.C.T. (VWR). Samples for histological staining were fixed in formalin for 24 hours and then stored in $70 \%$ ethanol before being embedded in paraffin. Human kidney samples were received embedded in paraffin. Paraffin-embedded tissues were cut into 4- to 6- $\mu \mathrm{m}$ sections and stained with Masson's trichrome and picrosirius red. Images were captured on a Carl Zeiss AxioImager.M2 using AxioVision SE64 software by Plan Apochromat $20 \times / 0.8$ objective. All images were analyzed through NIH ImageJ using a color threshold algorithm (identical threshold settings for compared image sets) written by Gabriel Landini (version v1.8) available at http://www.mecourse.com/landinig/software/software.html.

Immunofluorescence and quantitative microscopy. OCT-embedded mouse kidneys and paraffin-embedded human kidneys were cut into 4 - to $6-\mu \mathrm{m}$ sections and permeabilized in $1 \times$ PBS containing Triton X-100 $(0.1 \%)$ for 10 minutes. The sections were then labeled with Cy3- $\alpha$ SMA (1:500; Cell Signaling Technology, C6198), aSMA-FITC (1:500; Sigma-Aldrich, F3777), and anti-SMOC2 (1/250; Santa Cruz Biotechnology Inc., sc-67396). Slides with anti-SMOC2 were subsequently exposed to donkey anti-rabbit specific Cy3-conjugated secondary antibodies (1:500; Jackson ImmunoResearch Laboratories, 711-165-152). 4,6-Diamidino-2-phenylindole (Sigma-Aldrich) was used for nuclear staining (blue). Confocal images were acquired in the Nikon Imaging Center at Harvard Medical School. Images were collected with a Yokogawa CSU-X1 spinning disk confocal with Borealis modification, mounted on a Nikon Ti inverted microscope equipped with 20×/0.75 Plan Apo, 40×/1.3 Plan Fluor, 60×/1.4 Plan Apo objective lens, a Prior Proscan II motorized stage, and the Nikon Perfect Focus System for continuous maintenance of focus. FITC fluorescence was excited with an AOTF-controlled $488 \mathrm{~nm}$ solid state laser and collected with a 525/50 emission filter (Chroma). Cy3 fluorescence was excited with an AOTF-controlled $561 \mathrm{~nm}$ solid-state laser and collected with a 620/60 emission filter (Chroma). For both FITC and Cy3, a Quad 405/491/561/642 dichroic mirror was used (Semrock). DAPI was excited using a Lumencor SOLA with a 395/25 excitation filter, and emission was collected through the spinning disc head using a 460/25 emission filter. Images were acquired with a Hamamatsu ORCAAG cooled CCD camera controlled with MetaMorph 7 software. Brightness and contrast were adjusted on displayed images (identically for compared image sets) and quantified (identical threshold settings for compared image sets) using MetaMorph 7 software. 


\section{Western blot analysis}

Kidney tissues and cell cultures were homogenized in RIPA buffer (Thermo Fisher Scientific, $50 \mathrm{mM}$ Tris$\mathrm{HCl}$ [pH 7.4], $150 \mathrm{mM} \mathrm{NaCl}, 1 \% \mathrm{NP} 40$ ) containing $1 \times$ protease and phosphatase inhibitor cocktail (Roche Applied Science). Protein concentrations were determined using the BCA protein estimation kit (Pierce), and an equal amount of protein $(25 \mu \mathrm{g})$ was loaded on either a $10 \%$ or $12 \%$ polyacrylamide gel (PAGE). Protein transfer was performed using a nitrocellulose membrane. The following primary antibodies were used to detect the specific protein: anti-SMOC2 (1/250; Santa Cruz Biotechnology Inc., sc-67396), anti- $\alpha$ SMA (1/1,000; Sigma-Aldrich, A2547), anti-Collagen 1 $\alpha 1$ (1:250; Novus, NB600-408), anti-Fibronectin (1:250; Abcam, ab23750), anti-GAPDH (1/5,000; Abcam, ab181602), anti-MLC2-P (Thr18/Ser19) (1/1,000; Cell Signaling Technology, 3674), anti-Pax-P (Tyr118) (1/1,000; Cell Signaling Technology, 2541), anti-Phospho-FAK (Tyr925) (1/1,000; Cell Signaling Technology, 3284), MYC (1/5000; Sigma-Aldrich, M4439) and Integrin $\beta 1$ (1/1000; Santa Cruz, sc-8978). Horseradish peroxidase-conjugated secondary antibodies against mouse (Cell Signaling Technology, 7076) and rabbit (Cell Signaling Technology, 7074) were used to detect the appropriate primary antibody. Bands were detected with enhanced chemiluminescence (ECL) method (Pierce) and captured with Gel Doc XR+ System (Bio-Rad).

\section{Quantitative PCR}

Total RNA was isolated from cell cultures or tissue samples using TRIzol (Invitrogen) according to the manufacturer's protocol. RNA concentration was measured using a NanoDrop spectrophotometer (Thermo Fisher Scientific). Isolated RNA (1 $\mu \mathrm{g})$ was reverse transcribed into cDNA using a QuantiTect Reverse Transcription kit from Qiagen. Quantitative PCR (qPCR) was performed using a QuantiFast SYBR Green PCR kit (Qiagen) on a QuantStudio7 (Applied Biosystems) with the following thermal profile: activation $15 \mathrm{~s}$ at $95^{\circ} \mathrm{C} ; 40$ cycles of annealing/elongation $15 \mathrm{~s}$ at $94^{\circ} \mathrm{C}, 30 \mathrm{~s}$ at $60^{\circ} \mathrm{C}$; extension $30 \mathrm{~s}$ at $72^{\circ} \mathrm{C}$. All samples were measured with technical duplicates and normalized against GAPDH. Changes in the mRNA expression were calculated using the $\Delta \Delta \mathrm{Ct}$ method relative to a control. Forward and reverse primer sequences for mouse-specific genes are listed in Supplemental Table 2.

\section{RNA sequencing}

Library preparation. RNA samples ( $n=3-4$ mice/time point/group) were checked for quality and quantity using nanodrop and Agilent Bioanalyzer instrument. All RNA samples had RNA integrity numbers (RIN) higher than 7. Libraries were prepared using a TruSeq Stranded mRNA Library Prep Kit (Illumina) following the manufacturer's protocol modified as follows: For each sample, $330 \mathrm{ng}$ of RNA was input with $6.67 \mu 1$ of 1:1,000 ERCC spike-in Mix 2 (Ambion), fragmentation was done for 8 minutes, and 13 PCR cycles was used for the final library amplification. The finished dsDNA libraries were quantified by Qubit fluorometer, Agilent TapeStation 2200, and qPCR using the Kapa Biosystems library quantification kit according to manufacturer's protocols. Uniquely indexed libraries were pooled in equimolar ratios and sequenced on a single Illumina NextSeq500 run with single-end 75 bp reads by the Dana-Farber Cancer Institute Molecular Biology Core Facilities (Boston, MA). STAR aligner was used to map sequenced reads to build 9 of the mus musculus genome (mm9) genome assembly and to quantify gene level expression. The full dataset is available in the NCBI GEO database with the accession number GSE85209.

Bioinformatics analysis. All samples were processed using an RNA-seq pipeline implemented in the bcbio-nextgen project (https://bcbio-nextgen.readthedocs.org/en/latest/). Raw reads were examined for quality issues using FastQC (https://www.bioinformatics.babraham.ac.uk/projects/fastqc/) to ensure library generation and sequencing were suitable for further analysis. Adapter sequences, other contaminant sequences such as polyA tails, and low-quality sequences with Phred quality scores less than 5 were trimmed from reads using cutadapt (37). Trimmed reads were aligned to UCSC mm10, augmented with transcript information from Ensembl release GRCm38 using the STAR aligner (38). Alignments were checked for evenness of coverage, ribosomal RNA (rRNA) content, genomic context of alignments (for example, alignments in known transcripts and introns), complexity, and other quality checks using a combination of FastQC, Qualimap (39), and custom scripts. Counts of reads aligning to known genes were generated by featureCounts (40). Differential expression at the gene level was called with DESeq2 (41). DESeq2 was used to find how the two genotypes reacted differently to treatment using the Wald significance test and formula designed to find the difference in differences, or the intersection term between genotype and treatment in this DESeq2 design formula: genotype+Treatment+genotype:Treatment. As a result of this approach, fold-change values 
describe the differential effect of genotype on expression changes after treatment, not the direct gene expression, which would be observed directly between two sample classes. PCA analysis was performed on DESeq2 normalized, rlog variance stabilized reads. A cut-off-free GSEA for GO and KEGG terms was performed on the fold-change values derived from DESeq2 using GAGE (42) and visualized with REVIGO (43) treemaps. Expression patterns of genes within enriched GO terms were visualized by heatmap, after centering and scaling each genotype's expression values to their respective untreated sample's mean expression values (each sample's expression value was subtracted from the mean expression value for the sample genotype's untreated samples and divided by the mean's associated standard deviation).

Cell culture, reagents, and in vitro assays

In vitro cell culture. Primary human kidney fibroblasts were purchased from Cell Biologics and NIH3T3 cells from ATCC, which were grown as a monolayer in polystyrene culture dishes containing DMEM: Nutrient Mixture F-12 (DMEM/F12) (Invitrogen Corporation) supplemented with 10\% FBS (Invitrogen Corporation). Cells were grown until $80 \%$ confluency before passage. HPTECs were purchased from Biopredic and cultured in DMEM/F12 supplemented with hydrocortisone, EGF, insulin, transferrin, and sodium selenite. Cells were maintained at $37^{\circ} \mathrm{C}$ in a humidified $5 \% \mathrm{CO}_{2}$ incubator. For experiments studying FMT (44), fibroblasts were cultured in DMEM/F12 10\% FBS at low density for 24 hours, then at 40\%-50\% confluency, media was changed to DMEM/F12 1\% FBS for 4 hours prior to treatments with SMOC2 (Preprotech) and/or TGF $\beta 1$ (Preprotech). For antibody competition studies, recombinant SMOC2 was preincubated with human SMOC2 antibody (R\&D Systems, MAB5140) or human/mouse SMOC2 antibody (R\&D Systems, AF5140) and placed on rotation at room temperature for 1 hour, prior to being treated on cells. Integrin $\beta 1$ (Santa Cruz Biotechnology Inc., sc-8978) antibody was preincubated with cells at $37^{\circ} \mathrm{C}$ prior to SMOC2 treatment. Cell lines were not reported in the ICLAC database of commonly misidentified cell lines.

Transfections. For SMOC2-knockdown experiments, NIH3T3 fibroblasts were transfected with $100 \mathrm{nM}$ scramble or SMOC2 siRNA (Dharmacon) with siPORT NeoFX transfection reagent (Invitrogen) following the manufacturer's protocol. After 24 hours in DMEM/F12 10\%FBS, cells were harvested for Western blot analysis or were treated with trypsin and reseeded at 40\% confluency in cases of TGF $\beta 1$ stimulation. For overexpression studies, NIH3T3 cells were transfected with either pCMV Myc (pCMV), SMOC2 (MycDDK-tagged) - Human SPARC (pCMV-SMOC2), or MGP (GFP-tagged) - Human matrix Gla protein (MGP) plasmids (Origene Technologies) with Lipofectamine 2000 (Invitrogen) following the manufacturer's protocol. After 24 hours in DMEM/F12 10\%FBS, cells were harvested for Western blot and reverse transcription PCR analysis or were treated with trypsin and reseeded at $40 \%$ confluency for various assays.

Immunoprecipitation analysis. Cells were homogenized in RIPA buffer (Thermo Fisher Scientific, 50 $\mathrm{mM}$ Tris- $\mathrm{HCl}$ [pH 7.4], $150 \mathrm{mM} \mathrm{NaCl}, 1 \% \mathrm{NP} 40$ ) containing $1 \times$ protease and phosphatase inhibitor cocktail (Roche Applied Science) and left on a rocking platform for 1 hour. Protein concentration was determined using pierce BCA protein assay (Thermo Fisher Scientific). Between 500 $\mathrm{gg}$ to $1 \mathrm{mg}$ of protein was incubated with anti-MYC beads (Sigma-Aldrich) or Integrin $\beta 1$ antibody with Protein $\mathrm{G}$ beads (Sigma-Aldrich) for 4 hours at $4^{\circ} \mathrm{C}$. After centrifugation, the supernatant was removed and the beads were washed 5 times with $500 \mu$ of wash buffer ( $50 \mathrm{mM}$ TRIS buffer, $100 \mathrm{mM} \mathrm{NaCl}, 0.5 \%$ NP-40 and $1 \mathrm{mM}$ DTT). The beads were then boiled in $1 \times$ SDS solution for 10 minutes, followed by centrifugation, and used for Western blot analysis.

Immunofluorescence. NIH3T3 cells were fixed with 4\% paraformaldehyde (Thermo Fisher Scientific) in PBS, permeabilized with $0.1 \%$ Triton X-100 (Thermo Fisher Scientific) in PBS, and then blocked in 3\% BSA (Sigma-Aldrich). Cytoskeletal F-Actin was visualized using Alexa Fluor 564-conjugated Rhodamine Phalloidin (Thermo Fisher Scientific) at 1:500 in PBS for 1 our. 4,6-Diamidino-2-phenylindole (Sigma-Aldrich) was used for nuclear staining (blue). Confocal images were acquired in the Nikon Imaging Center at Harvard Medical School as described above.

Scratch assay. Fibroblasts were grown to a semiconfluent monolayer and then, in DMEM/F12 1\% FBS, were mechanically scratched (wound) using a standard $200 \mu 1$ pipette tip. Suspension cells were washed away with DMEM/F12 1\% FBS. Along the scratch, prefixed points were selected for representative photographs at 0 hours and 24 hours after initialization of the wound using a phase-contrast microscope. Wound closure was calculated by the percentage of newly covered area of SMOC2-treated fibroblasts over normal during 24 hours ( $n=5,3$ images per sample). Distance migrated from untreated cells was taken as $100 \%$. Representative images have been stained with methylene blue at 24 hours for increased contrast. 
MTT assay. NIH3T3 cells (7,500 cells) were plated in a 96-well plate for 24 hours, after which they were serum deprived in DMEM/F12 0.5\% FBS. Fibroblasts were then treated with different concentrations of SMOC2 for 24, 48, 72, and 96 hours in $0.2 \%$ FBS. To each sample, $1 \mathrm{mg} / \mathrm{ml}$ MTT was added 2 hours prior to each time point. The medium was aspirated, and $100 \mathrm{ml}$ isopropanol was added. Absorbance was measured at $570 \mathrm{~nm}$, taking $630 \mathrm{~nm}$ as a reference using SpectraMax Paradigm (Molecular Devices). Absorbance obtained from untreated cells was taken as $100 \%$ ( $n=5$ per concentration per time point).

Boyden chamber assay. Serum free media in the presence and absence of treatments were added in the lower chamber of a Chemotaxis Cell Migration Assay, 96-well $(8 \mu \mathrm{m})$ plate (Millipore). NIH3T3 cells were grown in 10\% FBS for 24 hours before being plated in $0.2 \%$ FBS of the upper migration chamber of an 8 $\mu \mathrm{M}$ 96-well plate for 24 hours. The migration assay was performed following the manufacturer's protocol.

Cell adhesion assay. NIH3T3 cells (7,500 cells) were plated in a 96-well plate for 24 hours. Cells were harvested with trypsin and reseeded into 96 -well plates at $37^{\circ} \mathrm{C}$. After 1 hour incubation, unattached cells were removed by $2 \times$ PBS washes. Adherent fibroblasts were fixed with methanol and stained with $1 \%$ crystal violet. Absorbance was measured using SpectraMax Paradigm (Molecular Devices). Absorbance obtained from untreated cells was taken as $100 \%(n=3)$.

\section{Statistics}

Data are expressed as the average \pm SEM. Statistical significance for multiple comparisons was evaluated by one-way ANOVA with Tukey post-hoc analysis $(P<0.05)$, using GraphPad Prism (GraphPad software). Statistical significance for single comparisons was calculated by two-tailed Student's $t$ test $(P<0.05)$, using Microsoft Excel. The sample size was predetermined based on the effect size and variability observed previously from similar readouts in our laboratory.

\section{Study approval}

The IRB at the Harvard Medical School approved the protocols for recruitment and urine sample collection, which was performed with written informed consent of the participants. All animal maintenance and treatment protocols were in compliance with the Guide for the Care and Use of Laboratory Animals (National Academies Press, 2011) as adopted and promulgated by the NIH and were approved by the Harvard Medical School Animal Care and Use Committees.

\section{Author contributions}

CG and VSV conceived and designed this study; CG, RKK, and AKA performed in vivo experiments; CG performed in vitro experiments; PT and AI assisted in specific parts of in vitro and in vivo experiments; SB made libraries for RNA sequencing; JNH performed bioinformatics to analyze RNA sequencing data; SSW provided human biospecimens and analyzed human data; CG and VSV analyzed and interpreted all the data; and CG and VSV wrote the manuscript. All authors provided constructive comments and edits, and approved the final version of this manuscript.

\section{Acknowledgments}

We thank Jennifer Waters and Talley Lambert of the Harvard Medical School's Nikon Imaging Center for their help in quantitative microscopy. We thank Zach Herbert at the Dana-Farber Cancer Institute's Molecular Biology Core Facility for his help in RNA sequencing. We also thank Vanesa Bijol, Department of Pathology, BWH, for providing deidentified human kidney tissue samples from patients with severe kidney fibrosis. Work in the Vaidya laboratory was supported by Outstanding New Environmental Sciences award from NIH/NIEHS (ES017543) and Innovation in Regulatory Science Award from Burroughs Wellcome Fund (BWF-1012518) and Partners Innovation Discovery Grant. Work by JNH was supported by funding from Harvard Catalyst | The Harvard Clinical and Translational Science Center (NIH award UL1 RR 025758 and financial contributions from participating institutions) and award number P30 ES00002 from the National Institute of Environmental Health Sciences. SB is supported by the Harvard Medical School Center of Excellence in Systems Pharmacology NIH grant P50 GM107618 and the Giovanni Armenise-Harvard Foundation.

Address correspondence to: Vishal S. Vaidya, Harvard Institutes of Medicine, Room 562, 77 Avenue Louis Pasteur, Boston, Massachusetts 02115, USA. Phone: 617.525.5974; E-mail: vvaidya@bwh.harvard.edu. 
1. Nanthakumar CB, Hatley RJ, Lemma S, Gauldie J, Marshall RP, Macdonald SJ. Dissecting fibrosis: therapeutic insights from the small-molecule toolbox. Nat Rev Drug Discov. 2015;14(10):693-720.

2. Ferenbach DA, Bonventre JV. Mechanisms of maladaptive repair after AKI leading to accelerated kidney ageing and CKD. Nat Rev Nephrol. 2015;11(5):264-276.

3. Breyer MD, Susztak K. The next generation of therapeutics for chronic kidney disease. Nat Rev Drug Discov. 2016;15(8):568-588.

4. Grgic I, Duffield JS, Humphreys BD. The origin of interstitial myofibroblasts in chronic kidney disease. Pediatr Nephrol. 2012;27(2):183-193.

5. Duffield JS. Cellular and molecular mechanisms in kidney fibrosis. J Clin Invest. 2014;124(6):2299-2306.

6. Tomasek JJ, Gabbiani G, Hinz B, Chaponnier C, Brown RA. Myofibroblasts and mechano-regulation of connective tissue remodelling. Nat Rev Mol Cell Biol. 2002;3(5):349-363.

7. Hinz B. It has to be the $\alpha v$ : myofibroblast integrins activate latent TGF- $\beta 1$. Nat Med. 2013;19(12):1567-1568.

8. Meran S, Steadman R. Fibroblasts and myofibroblasts in renal fibrosis. Int J Exp Pathol. 2011;92(3):158-167.

9. LeBleu VS, et al. Origin and function of myofibroblasts in kidney fibrosis. Nat Med. 2013;19(8):1047-1053.

10. Craciun FL, et al. RNA Sequencing Identifies Novel Translational Biomarkers of Kidney Fibrosis. J Am Soc Nephrol. 2016;27(6):1702-1713.

11. Wong GS, Rustgi AK. Matricellular proteins: priming the tumour microenvironment for cancer development and metastasis. $\mathrm{Br}$ J Cancer. 2013;108(4):755-761.

12. Schellings MW, Pinto YM, Heymans S. Matricellular proteins in the heart: possible role during stress and remodeling. Cardiovasc Res. 2004;64(1):24-31.

13. Jendraschak E, Sage EH. Regulation of angiogenesis by SPARC and angiostatin: implications for tumor cell biology. Semin Can cer Biol. 1996;7(3):139-146.

14. Craciun FL, et al. Pharmacological and genetic depletion of fibrinogen protects from kidney fibrosis. Am J Physiol Renal Physiol. 2014;307(4):F471-F484.

15. Yuan HT, Li XZ, Pitera JE, Long DA, Woolf AS. Peritubular capillary loss after mouse acute nephrotoxicity correlates with down-regulation of vascular endothelial growth factor-A and hypoxia-inducible factor-1 alpha. Am J Pathol. 2003;163(6):2289-2301.

16. Long DA, Woolf AS, Suda T, Yuan HT. Increased renal angiopoietin-1 expression in folic acid-induced nephrotoxicity in mice. J Am Soc Nephrol. 2001;12(12):2721-2731.

17. Surendran K, Simon TC, Liapis H, McGuire JK. Matrilysin (MMP-7) expression in renal tubular damage: association with Wnt4. Kidney Int. 2004;65(6):2212-2222.

18. Kang HM, et al. Defective fatty acid oxidation in renal tubular epithelial cells has a key role in kidney fibrosis development. Nat Med. 2015;21(1):37-46.

19. Kang HM, Huang S, Reidy K, Han SH, Chinga F, Susztak K. Sox9-Positive Progenitor Cells Play a Key Role in Renal Tubule Epithelial Regeneration in Mice. Cell Rep. 2016;14(4):861-871.

20. Chevalier RL, Forbes MS, Thornhill BA. Ureteral obstruction as a model of renal interstitial fibrosis and obstructive nephropathy. Kidney Int. 2009;75(11):1145-1152.

21. Yang L, Besschetnova TY, Brooks CR, Shah JV, Bonventre JV. Epithelial cell cycle arrest in G2/M mediates kidney fibrosis after injury. Nat Med. 2010;16(5):535-543.

22. Maier S, Paulsson M, Hartmann U. The widely expressed extracellular matrix protein SMOC-2 promotes keratinocyte attachment and migration. Exp Cell Res. 2008;314(13):2477-2487.

23. Mitra SK, Hanson DA, Schlaepfer DD. Focal adhesion kinase: in command and control of cell motility. Nat Rev Mol Cell Biol. 2005;6(1):56-68.

24. Mimura Y, Ihn H, Jinnin M, Asano Y, Yamane K, Tamaki K. Constitutive phosphorylation of focal adhesion kinase is involved in the myofibroblast differentiation of scleroderma fibroblasts. J Invest Dermatol. 2005;124(5):886-892.

25. Greenberg RS, Bernstein AM, Benezra M, Gelman IH, Taliana L, Masur SK. FAK-dependent regulation of myofibroblast differentiation. FASEB J. 2006;20(7):1006-1008

26. Gerarduzzi C, He Q, Antoniou J, Di Battista JA. Prostaglandin E (2)-dependent blockade of actomyosin and stress fibre formation is mediated through S1379 phosphorylation of ROCK2. J Cell Biochem. 2014;115(9):1516-1527.

27. Chau BN, et al. MicroRNA-21 promotes fibrosis of the kidney by silencing metabolic pathways. Sci Transl Med. 2012;4(121):121ra18.

28. Humphrey JD, Dufresne ER, Schwartz MA. Mechanotransduction and extracellular matrix homeostasis. Nat Rev Mol Cell Biol. 2014;15(12):802-812.

29. McCurdy S, Baicu CF, Heymans S, Bradshaw AD. Cardiac extracellular matrix remodeling: fibrillar collagens and Secreted Protein Acidic and Rich in Cysteine (SPARC). J Mol Cell Cardiol. 2010;48(3):544-549.

30. Sasaki T, Hohenester E, Göhring W, Timpl R. Crystal structure and mapping by site-directed mutagenesis of the collagen-binding epitope of an activated form of BM-40/SPARC/osteonectin. EMBO J. 1998;17(6):1625-1634.

31. Bradshaw AD, et al. Pressure overload-induced alterations in fibrillar collagen content and myocardial diastolic function: role of secreted protein acidic and rich in cysteine (SPARC) in post-synthetic procollagen processing. Circulation. 2009;119(2):269-280.

32. Pazin DE, Albrecht KH. Developmental expression of Smoc1 and Smoc2 suggests potential roles in fetal gonad and reproductive tract differentiation. Dev Dyn. 2009;238(11):2877-2890.

33. Rocnik EF, Liu P, Sato K, Walsh K, Vaziri C. The novel SPARC family member SMOC-2 potentiates angiogenic growth factor activity. J Biol Chem. 2006;281(32):22855-22864.

34. Liu P, Lu J, Cardoso WV, Vaziri C. The SPARC-related factor SMOC-2 promotes growth factor-induced cyclin D1 expression and DNA synthesis via integrin-linked kinase. Mol Biol Cell. 2008;19(1):248-261.

35. Bornstein P, Sage EH. Matricellular proteins: extracellular modulators of cell function. Curr Opin Cell Biol. 2002;14(5):608-616.

36. Grinnell F. Fibroblast biology in three-dimensional collagen matrices. Trends Cell Biol. 2003;13(5):264-269. 
37. Martin M. Cutadapt removes adapter sequences from high-throughput sequencing reads. Embnet.journal. $2011 ; 17(1): 10-12$.

38. Dobin A, et al. STAR: ultrafast universal RNA-seq aligner. Bioinformatics. 2013;29(1):15-21.

39. García-Alcalde F, et al. Qualimap: evaluating next-generation sequencing alignment data. Bioinformatics. 2012;28(20):2678-2679.

40. Liao Y, Smyth GK, Shi W. featureCounts: an efficient general purpose program for assigning sequence reads to genomic features. Bioinformatics. 2014;30(7):923-930.

41. Love MI, Huber W, Anders S. Moderated estimation of fold change and dispersion for RNA-seq data with DESeq2. Genome Biol. 2014;15(12):550.

42. Luo W, Friedman MS, Shedden K, Hankenson KD, Woolf PJ. GAGE: generally applicable gene set enrichment for pathway analysis. BMC Bioinformatics. 2009;10:161.

43. Supek F, Bošnjak M, Škunca N, Šmuc T. REVIGO summarizes and visualizes long lists of gene ontology terms. PLoS One. 2011;6(7):e21800

44. Masur SK, Dewal HS, Dinh TT, Erenburg I, Petridou S. Myofibroblasts differentiate from fibroblasts when plated at low density. Proc Natl Acad Sci USA. 1996;93(9):4219-4223. 